\title{
Causal Networks and Freedom of Choice in Bell's Theorem
}

\author{
Rafael Chaves $\odot, 1,2,{ }^{*}$ George Moreno $\odot,{ }^{1}$ Emanuele Polino $\odot,{ }^{3}$ Davide Poderini, ${ }^{3}$ Iris Agresti, ${ }^{3}$ \\ Alessia Suprano, ${ }^{4}$ Mariana R. Barros $\odot,{ }^{4}$ Gonzalo Carvacho, ${ }^{3}$ Elie Wolfe $\odot,{ }^{6}$ Askery Canabarro $\odot,{ }^{1,6}$ \\ Robert W. Spekkens, ${ }^{5}$ and Fabio Sciarrino ${ }^{3}$ \\ ${ }^{1}$ International Institute of Physics, Federal University of Rio Grande do Norte, P.O. Box 1613, Natal 59078-970, \\ Brazil \\ ${ }^{2}$ School of Science and Technology, Federal University of Rio Grande do Norte, Natal 59078-970, Brazil \\ ${ }^{3}$ Dipartimento di Fisica-Sapienza Università di Roma, Piazzale Aldo Moro 5, Roma I-00185, Italy \\ ${ }^{4}$ Dipartimento di Fisica “Ettore Pancini," Università Federico II, Complesso Universitario di Monte \\ Sant'Angelo, Via Cintia, Napoli 80126, Italy \\ ${ }^{5}$ Perimeter Institute for Theoretical Physics, 31 Caroline Street North, Waterloo, Ontario N2L 2Y5, Canada \\ ${ }^{6}$ Grupo de Física da Matéria Condensada, Núcleo de Ciências Exatas-NCEx, Campus Arapiraca, Universidade \\ Federal de Alagoas, Arapiraca-AL 57309-005, Brazil
}

(Received 28 May 2021; accepted 23 September 2021; published 3 November 2021)

\begin{abstract}
Bell's theorem is typically understood as the proof that quantum theory is incompatible with localhidden-variable models. More generally, we can see the violation of a Bell inequality as witnessing the impossibility of explaining quantum correlations with classical causal models. The violation of a Bell inequality, however, does not exclude classical models where some level of measurement dependence is allowed, that is, the choice made by observers can be correlated with the source generating the systems to be measured. Here, we show that the level of measurement dependence can be quantitatively upper bounded if we arrange the Bell test within a network. Furthermore, we also prove that these results can be adapted in order to derive nonlinear Bell inequalities for a large class of causal networks and to identify quantumly realizable correlations that violate them.
\end{abstract}

DOI: 10.1103/PRXQuantum.2.040323

\section{INTRODUCTION}

Bell's theorem [1] can arguably be seen as the most radical departure from classical physics. The kind of nonclassicality it entails is achieved without the need of any specific details or experimental assumptions that, furthermore, can be put to practical use in a variety of quantum information processing protocols in what is known as the device-independent framework [2].

In its standard interpretation, the violation of a Bell inequality shows that quantum correlations are incompatible with any theories respecting local realism, that is, theories where physical properties have well-defined values prior to any measurement and in such a way that faraway events do not have a direct causal influence over each

\footnotetext{
*rchaves@iip.ufrn.br
}

Published by the American Physical Society under the terms of the Creative Commons Attribution 4.0 International license. Further distribution of this work must maintain attribution to the author(s) and the published article's title, journal citation, and DOI. other. From another perspective, Bell-inequality violation can also be seen as disproving any theories obeying the notion of local causality, which implies a certain factorization of probabilities, and that can be derived from two more fundamental assumptions, i.e., Reichenbach's principle [3] and relativistic causality, according to which the past corresponds to the past light cone (see Refs. [4,5]). However, as first pointed out by Brans [6], local-hidden-variable models are still capable of reproducing the quantum predictions if we allow for measurement dependence, a mechanism where our measurement devices are correlated with the system to be measured (see also Refs. [7-9]). This subtle assumption in Bell's theorem, also known as the assumption of "free will" or as "statistical independence," has since then attracted growing attention, both from a theoretical [9-21] and experimental [22-25] perspective, and can be related to the communication cost between the measurement stations, needed by classical models to reproduce quantum correlations [11,26-34].

Of particular relevance is the paradigmatic ClauserHorne-Shimony-Holt (CHSH) Bell scenario [35]involving two distant parties, each measuring two dichotomic observables - which has been thoroughly 
analyzed also allowing for relaxations of the measurementdependence assumption [20]. As compared with the relaxation of locality, where it is known [29] that one requires 1 bit of classical communication to simulate the maximal quantum violation of the $\mathrm{CHSH}$ inequality, measurement dependence turns out to be a stronger resource, as merely 0.046 bits of correlation are already enough to achieve the simulation [20]. In spite of steady progress, all results to date suffer from the fact that they only impose lower bounds on the amount of measurement dependence needed to simulate a given violation of a Bell inequality using a classical causal model. If, by considering a slight modification of a Bell experiment, one had the means of determining an upper bound on the amount of measurement dependence that can be present, then whenever this amount was less than the lower bound on the amount needed to explain the observed violation of a Bell inequality in a classical causal model, one could infer that these violations were due to nonclassical effects. Such a modification, in other words, would provide a means of adjudicating between measurement dependence and nonclassicality as a means of explaining the violation. Measurement dependence has then remained as a seemingly untestable loophole in any Bell experiment.

The first aim of this paper is to revisit measurement dependence and show that under some assumptions, it can indeed be upper bounded from the data observed in a slightly modified Bell experiment. For that, we arrange the standard Bell scenario as part of a larger causal network that includes an auxiliary variable and use the correlations between the measurement inputs and the auxiliary variable in order to upper bound how much such inputs might depend on the source generating the physical systems to be measured. From that, we obtain nonlinear Bell inequalities - which explicitly incorporate possible correlations between the system to be measured and the settings of the measurement devices - the violation of which is a clear signature of nonclassicality, rather than something that can be explained by merely positing measurement dependence within a classical causal model.

Following this, we explore the connections between measurement-dependent Bell causal structures and general causal networks that have recently started to attract attention in the literature [36]. As compared with usual Bell scenarios, these new networks have two characteristic features: first, the fact that the correlations between the distant parties are now mediated by a number of independent sources; and, second, the fact that one can prove nonclassical behavior even without the need for any inputs, something considered quintessential in Bell's theorem [37]. In spite of the promising foundational and applied uses, progress in the analysis of general causal networks has been impeded by the fact that the correlations compatible with them define nonconvex sets, for which decades of expertise gathered with the standard Bell scenario and convex optimization algorithms are of limited or no use. Here, we show that standard Bell scenarios with measurement dependence can readily and generally be mapped onto causal networks of growing size and with different topologies. Not only do we derive new nonlinear Bell inequalities for a variety of networks but we also show, for the first time, that they lead to nonclassical correlations.

The paper is organized as follows. In Sec. II, we revisit the measurement-independence assumption in Bell's theorem from a causal perspective and, in particular, identify and discriminate two possible mechanisms able to generate measurement dependence. In Sec. III, we briefly present the entropic approach for the characterization of classical causal structures. In Sec. IV, we discuss and derive a number of results and inequalities for the characterization of bipartite Bell scenarios with measurement dependence. In this section, we also generalize these results to generic multipartite Bell scenarios and, in particular, derive strong lower bounds for measurement dependence based on the Mermin inequality [38]. In Sec. V, we adapt our results for the analysis of a wide variety of causal networks, in particular proving that they can lead to nonclassical correlations. Finally, in Sec. VI, we discuss our results and point out interesting directions for future research.

\section{FREEDOM OF CHOICE IN BELL'S THEOREM}

Bell's theorem [1] is the prime example of the incompatibility of quantum predictions with those of classical causal models. From a modern perspective $[4,17]$, we impose a given causal structure on our quantum experiment and inquire whether causal models of classical origin can explain the observed empirical data, that is, the observed probability distribution. Different causal structures can be used to that aim [39-49] but the paradigmatic illustration involves two distant parties, Alice and Bob, that upon receiving physical systems from a common source, randomly decide which measurements to perform obtaining the corresponding outcomes. Their measurement choices are labeled by the variables $X$ and $Y$, while their outcomes are labeled by $A$ and $B$, for Alice and Bob, respectively. We note that random variables are typically denoted by uppercase letters and the values that these variables can assume by lowercase letters. For instance, $X$ would represent the input variable of Alice and $X=x$, a specific value of it. In order to simplify the notation and presentation, in what follows we use lowercase letters only.

Invoking special relativity, if Alice and Bob are spacelike separated, then the measurement choices of one should have no causal influence on the measurement outcomes of the other. These are the so-called no-signaling constraints. They imply that the probability distribution $p(a, b \mid x, y)$ 
observed in such an experiment should respect

$$
\begin{aligned}
& p(a \mid x)=\sum_{b} p(a, b \mid x, y)=\sum_{b} p\left(a, b \mid x, y^{\prime}\right) \forall y, y^{\prime}, \\
& p(b \mid y)=\sum_{a} p(a, b \mid x, y)=\sum_{a} p\left(a, b \mid x^{\prime}, y\right) \forall x, x^{\prime} .
\end{aligned}
$$

From a causal-inference perspective [50], the central question is: what is the simplest causal structure able to recover, in a faithful manner, the probabilistic conditional independence relations corresponding to the no-signaling condition? That is, these conditional independencies should follow from the causal structure itself and not from a fine tuning of model parameters. More formally, a causal structure can be defined by a directed acyclic graph (DAG), where the nodes represent the variables of interest and direct edges encode the causal relations among them [50]. Classically, any node $x_{i}$ in the graph can be understood as a function of its graph-theoretical parents $\mathrm{Pa}\left(x_{i}\right)$, implying the causal Markov condition [50], which stipulates that a variable is conditionally independent of its nondescendents given its parents and thus

$$
p\left(x_{1}, \ldots, x_{n}\right)=\prod_{i=1, \ldots, n} p\left[x_{i} \mid \operatorname{Pa}\left(x_{i}\right)\right] .
$$

It turns out that [4] the observational equivalence class of causal structures that faithfully (that is, without fine tuning of parameters) imply the no-signaling constraints is the one that includes the causal structure posited by Bell in his seminal work, shown in Fig. 1 [51] Employing the causal Markov condition [50], any probability compatible with Bell's causal structure should fulfill

$$
p(a, b \mid x, y)=\sum_{\lambda} p(a \mid x, \lambda) p(b \mid y, \lambda) p(\lambda) .
$$

As shown by Bell [1], a quantum experiment that mirrors this causal structure can yield correlations that

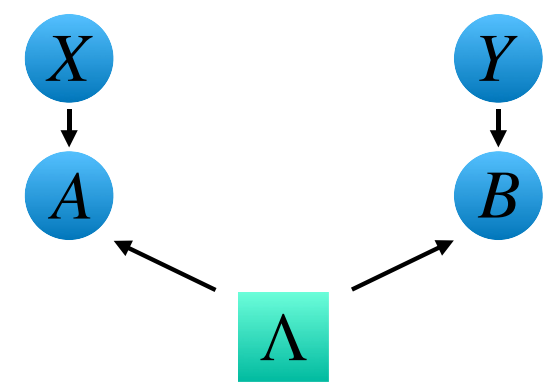

FIG. 1. Bell's causal structure. Two distant parties receive their shares of a joint subsystem prepared by a common source $\Lambda$. The variables $X$ and $Y$ represent the measurement choices and $A$ and $B$ the corresponding measurement outcomes for the observers Alice and Bob, respectively. are incompatible with the classical description given by Eq. (3). This is the phenomenon known as Bell nonlocality, which can be witnessed by violating Bell inequalities, linear constraints of the general form

$$
I=\sum_{a, b, x, y} \alpha_{a, b, x, y} p(a, b \mid x, y) \leq L,
$$

where $L$ is the maximum possible value achievable by the classical description given in Eq. (3).

It is often said that Bell's theorem shows the incompatibility between quantum theory and the assumptions of realism, locality, and freedom of choice. Under this standard view, the classical decomposition given in Eq. (3) is referred to as a local-hidden-variable (LHV) model. This interpretation follows naturally from the causal perspective. The realism assumption corresponds to the assumption of explainability of the correlations in terms of a classical causal model, in particular, one positing the existence of a hidden variable $\Lambda$. In turn, the locality and freedom-of-choice (or measurement-independence) assumptions are encoded in the causal structure of Fig. 1. Through the Markov condition, this structure implies that $p(a \mid b, y, x, \lambda)=p(a \mid x, \lambda)$ and $p(b \mid a, x, y, \lambda)=p(b \mid y, \lambda)$, a condition that is typically called 'local causality' [5,52] and that asserts that Alice's and Bob's outcomes are fully determined by their choices and the state of the source. It also implies that $p(x, y, \lambda)=p(x, y) p(\lambda)$, the condition that is typically taken to formalize the notion of measurement independence and that asserts that Alice's and Bob's choices are independent from the common source establishing the correlations.

\section{A. When measurement independence fails}

The failure of measurement independence is called measurement dependence. In a scenario with measurement dependence, we have $p(x, y, \lambda) \neq p(x, y) p(\lambda)$. In stark contrast with the causal models of the standard Bell scenario given by Eq. (3), in the presence of measurement dependence we allow $p(\lambda \mid x, y) \neq p(\lambda)$. Without measurement independence, the admissible correlations $p(a, b \mid x, y)$ are fairly unrestricted; certainly, standard Bell inequalities would no longer constrain them.

However, just because one grants that $X$ or $Y$ might be somewhat correlated with $\Lambda$ does not mean that $X$ and $Y$ are not also largely functions of causal factors that are independent of $\Lambda$. To this end, we formally introduce local-laboratory private randomness sources $U_{x}$ and $U_{y}$ for Alice and Bob, respectively, as depicted in Fig. 2. We now provide some physical intuition for why it makes sense to model $X$ and $Y$ in terms of both causal factors confounded with $\Lambda$ as well as private causal factors independent of $\Lambda$. 


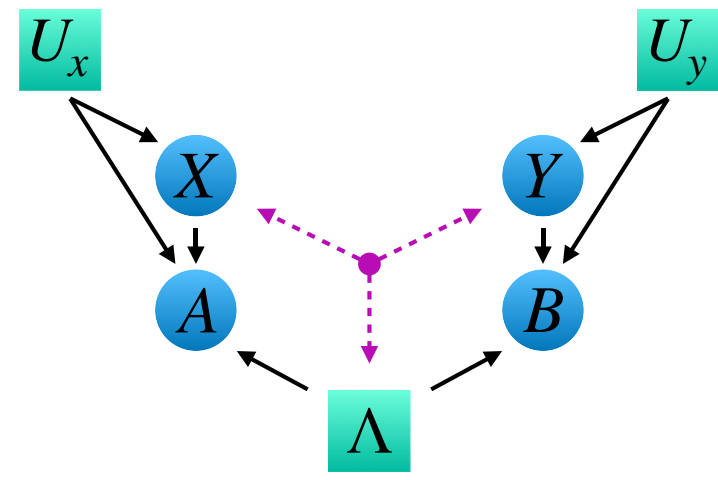

FIG. 2. Bell's causal structure with measurement dependence. The purple arrows represent the fact that the correlations between $X, Y$, and $\Lambda$ can be due to some direct causal influence among them or mediated by an external common source. We emphasize that even though the settings are plausibly common-cause connected with $\Lambda$, typically one can also be confident that they are also (strongly) influenced by independent localized sources of randomness.

Differently from the locality assumption that can be assured by invoking special relativity, measurement independence is a thorny issue. Nevertheless, the independence of $U_{x}, U_{y}$, and $\Lambda$ can indeed be made very plausible. For instance, $U_{x}$ and $U_{y}$ could denote stars emitting cosmic photons centuries ago $[23,25]$ or even the human randomness of hundreds of thousands of people around the globe [24]. It seems reasonable that such sources are independent of $\Lambda$, which in a photonic experiment would represent the laser and nonlinear crystal employed to generate entangled photons.

By explicitly introducing $U_{x}$ and $U_{y}$ into our causal models, we can now rephrase the assumption of measurement independence as equivalent to the assumption that $X$ depends exclusively on $U_{x}$ and not on any hidden factor correlated with $\Lambda$, i.e., the assumption that $p\left(x \mid u_{x}, \lambda\right)=p\left(x \mid u_{x}\right)$. While is clear that (by definition) $U_{x}$ is independent of $U_{y}$ and $\Lambda$, it can be difficult to rigorously justify the assumption of the independence of $X$ and $Y$ from $\Lambda$.

As an illustration for why this is the case, suppose that $U_{x}$ represents some faraway star emitting cosmic photons that define the variable $X$. There is no reason to doubt that this star is independent of a laser in a laboratory today. However, at some point, the photons emitted by these independent sources (the star and the laser) meet up within the physicist's laboratory and define the outcome $A$ they will give rise to. Within this context, the atmosphere in the laboratory (acting as a medium for the photons) or whatever else that might affect the photons state can act as a source of correlations and lead to deviations from perfect measurement independence.

In short, even though the experiment might use a source $U_{x}$ that is independent of $\Lambda$, a causal mediary between $U_{x}$ and the measurement outcome variable $A$ might nonetheless be influenced by something that also has an influence on $\Lambda$, so that $X$ and $\Lambda$ end up having a common cause (as illustrated in Fig. 2) and therefore the potential for a small amount of statistical dependence between them. Furthermore, as it turns out, even a quite small amount of measurement dependence (to be explicitly quantified below) is already sufficient to simulate the maximum possible Bell-inequality violation achievable with quantum mechanics [10,20].

Even though the sources $U_{x}, U_{y}$, and $\Lambda$ can be assumed to be independent, the most general LHV model represented by the DAG in Fig. 2 implies that

$$
\begin{aligned}
p(a, b, x, y)= & \sum_{\lambda}\left(\sum_{u_{x}} p\left(a \mid x, u_{x}, \lambda\right) p\left(x \mid u_{x}, \lambda\right) p\left(u_{x}\right)\right) \\
& \times\left(\sum_{u_{y}} p\left(b \mid y, u_{y}, \lambda\right) p\left(y \mid u_{y}, \lambda\right) p\left(u_{y}\right)\right) p(\lambda) \\
= & \sum_{\lambda} p(a \mid x, \lambda) p(x \mid \lambda) p(b \mid y, \lambda) p(y \mid \lambda) p(\lambda),
\end{aligned}
$$

such that

$$
\begin{aligned}
p(a, b \mid x, y)= & \sum_{\lambda}\left(\sum_{u_{x}} p\left(a \mid x, u_{x}, \lambda\right) p\left(u_{x} \mid x, \lambda\right)\right) \\
& \times\left(\sum_{u_{y}} p\left(b \mid y, u_{y}, \lambda\right) p\left(u_{y} \mid y, \lambda\right)\right) p(\lambda \mid x, y) \\
= & \sum_{\lambda} p(a \mid x, \lambda) p(b \mid y, \lambda) p(\lambda \mid x, y)
\end{aligned}
$$

Note that $p(\lambda \mid x, y)=p(\lambda)$ only if $p\left(x \mid u_{x}, \lambda\right)=p\left(x \mid u_{x}\right)$ and $p\left(y \mid u_{y}, \lambda\right)=p\left(y \mid u_{y}\right)$, in which case then we recover the standard measurement-independent model of Eq. (3).

\section{B. Quantifying measurement dependence}

In the special limit $p(x, y, \lambda)-p(x, y) p(\lambda) \rightarrow 0$, we recover measurement independence, so one natural measure for quantifying the degree of measurement dependence in a given Bell experiment is [17]

$$
\mathcal{M}:=\sum_{x, y, \lambda}|p(x, y, \lambda)-p(x, y) p(\lambda)|
$$

Clearly, when $\mathcal{M}=0$, the measurement-dependent model given in Eq. (6) goes over to the usual measurementindependent model, given in Eq. (3).

A related measure that has also been considered in the literature $[10,20]$ is the mutual information between the 
experimenter's choices and the source $\Lambda$, defined as

$$
I(X, Y: \Lambda):=H(X, Y)+H(\Lambda)-H(X, Y, \Lambda),
$$

where $H(X)=-\sum_{x} p(x) \log p(x)$ is the Shannon entropy of the random variable $X$. Importantly, the measures given in Eqs. (7) and (8) can be related via the Pinsker inequality [53]

$$
\mathcal{M}^{2} \leq \frac{I(X, Y: \Lambda)}{\log _{2} e}
$$

Within this context, we can then ask how much measurement dependence would be necessary to reproduce some Bell-inequality violation using the classical causal model described by Eq. (6) (see Fig. 2). For instance, in the paradigmatic Clauser-Horne-Shimony-Holt (CHSH) scenario [35], where each of the parties can measure two possible dichotomic measurements, the LHV models given in Eq. (3) respect the inequality

$$
\mathrm{CHSH}=\left\langle A_{0} B_{0}\right\rangle+\left\langle A_{0} B_{1}\right\rangle+\left\langle A_{1} B_{0}\right\rangle-\left\langle A_{1} B_{1}\right\rangle \leq 2,
$$

where $\left\langle A_{x} B_{y}\right\rangle=\sum_{a, b=0,1}(-1)^{a+b} p(a, b \mid x, y)$ is the expectation value of Alice's and Bob's outcomes $A$ and $B$ conditioned on the inputs $x$ and $y$ (with all input and output variables taking values 0 or 1 ). For the measurementdependent models given in Eq. (6), one can prove that [17]

$$
(\mathrm{CHSH}-2) / 4 \leq \mathcal{M} \text {. }
$$

In turn, using the mutual information, it has been proven [20] that

$$
2-h\left(\frac{4-\mathrm{CHSH}}{8}\right)-\frac{4+\mathrm{CHSH}}{8} \log _{2} 3 \leq I(X, Y: \Lambda),
$$

where $h(x)$ is the binary entropy given by

$$
h(x)=-x \log _{2} x-(1-x) \log _{2}(1-x) .
$$

This implies, in particular, that even for the maximal quantum violation of $2 \sqrt{2}$ of the CHSH inequality, a measurement dependence as low as $I(X, Y: \Lambda)=0.046$ bits is already enough to reproduce the quantum predictions.

Equations (11) and (12) show that the violation of a Bell inequality can be explained by some nonzero degree of measurement dependence. The problem, however, is that whether or not there is such measurement dependence cannot be determined in a standard Bell experiment. In other words, one cannot know if the violation of a given Bell inequality is due to quantum effects or simply because the measurement-independence assumption is not

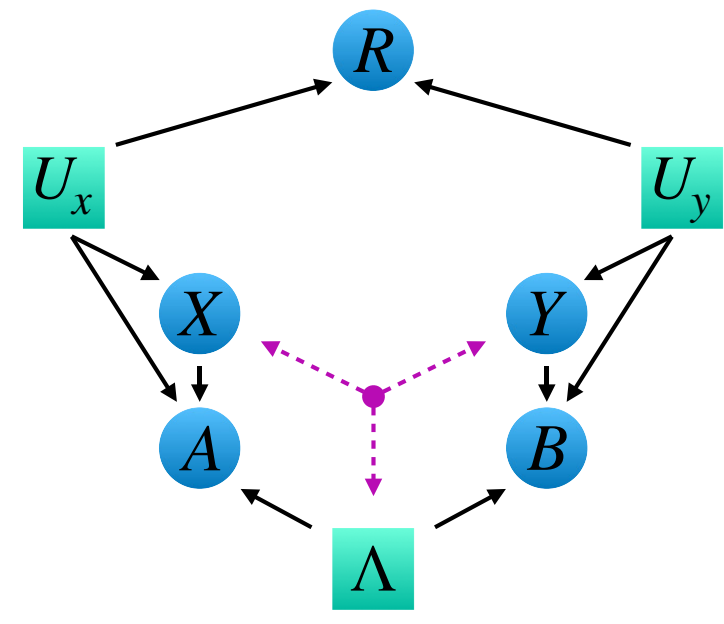

FIG. 3. A causal structure assessing the measurement dependence. The extra measurement outcome variable $R$ can be used to provide an upper bound on the measurement dependence. Note that this model allows, at least in principle, for arbitrary correlations between the input variables $X$ and $Y$ and the source $\Lambda$.

fulfilled. As such, the measurement-dependence loophole remains as a possible classical explanation for the violation of a Bell inequality, even for milestone experiments violating a Bell inequality, while sealing the locality- and detection-efficiency loopholes [54-56].

At the core of the problem is the fact that, in a standard Bell experiment, there is nothing that implies an upper bound on the amount of measurement dependence.

In the following, we show that by embedding a Bell experiment in a larger causal network that includes an auxiliary variable $R$ that is influenced by $U_{x}$ and $U_{y}$ but is independent of $\Lambda$ (see Fig. 3), we are able to derive an upper bound on the amount of measurement dependence. In essence, the idea is that, for this causal structure, if $R$ is strongly correlated with $X$, then $X$ must be only weakly correlated with $\Lambda$, and similarly for $Y$. (The limiting case of this trade-off- the fact that perfect correlation between $R$ and $X$ implies no correlation between $X$ and $\Lambda$-is what Fritz [41] has used to establish that the triangle causal scenario can be mapped onto the Bell scenario. The latter observation is therefore the root of the approach to bounding measurement dependence described in this paper.)

This fact allows us to answer in an unambiguous manner (assuming the sources $U_{x}, U_{y}$, and $\Lambda$ to be independent) the question of whether the violation of a Bell inequality is due to the presence of quantum entanglement or due to measurement dependence. We also extend our analysis to the multipartite case and show how our inequalities can be used to characterize a large class of causal networks that are increasingly attracting attention [39-49,57]. Prior to doing so, however, we briefly introduce the entropic 
framework for causal inference [58], which is crucial to deriving some of our technical results.

\section{ENTROPIC INEQUALITIES AND CAUSAL NETWORKS}

Our aim is to obtain an upper bound to the measurementdependence measures, $\mathcal{M}$ or $I(X, Y: \Lambda)$, in terms of the degree of correlation exhibited between $R$ and $X, Y$ in the causal structure of Fig. 3. Note that the joint distributions on observed variables that are compatible with the causal structure of Fig. 3 are of the form

$$
p(a, b, x, y, r)=\sum_{u_{x}, u_{y}, \lambda}\left(\begin{array}{l}
p\left(a, x \mid u_{x}, \lambda\right) p\left(b, y \mid u_{y}, \lambda\right) \times \\
p\left(r \mid u_{x}, u_{y}\right) p\left(u_{x}\right) p\left(u_{y}\right) p(\lambda)
\end{array}\right) .
$$

However, the fact that the sources $U_{x}, U_{y}$, and $\Lambda$ are independent implies that this set is nonconvex and therefore difficult to characterize $[44,59]$. This nonconvexity can be circumvented by the entropic approach introduced in Refs. [58,60-62], allowing one to obtain analytical bounds for $I(X, Y: \Lambda)$. The bounds can then also be translated into bounds on the L1-norm quantifier $\mathcal{M}$ via the Pinsker inequality [53].

A detailed account of the entropic approach can be found in Ref. [58]. Here, we introduce the central concepts necessary to understand the results that follow.

Consider a set of $n$ discrete random variables $X_{1}, \ldots, X_{n}$. We denote as $[n]=\{1, \ldots, n\}$ the set of indices of these random variables. For every subset $S \in$ $2^{[n]}$ of indices, $X_{S}$ is the random vector $\left(X_{i}\right)_{i \in S}$ and $H(S):=H\left(X_{S}\right)$ is its associated Shannon entropy, defined by $H\left(X_{S}\right):=-\sum_{x_{s}} p\left(x_{s}\right) \log _{2} p\left(x_{s}\right)$. We can construct an entropy vector $h=\left\{\varnothing, H\left(X_{1}\right), H\left(X_{2}\right), H\left(X_{1}, X_{2}\right), \ldots\right.$, $\left.H\left(X_{1}, \ldots, X_{n}\right)\right\}$ with all possible $2^{n}$ entropies for $n$ variables (including the empty set) and ask what the constraints are for $h$ to be a valid entropy vector. The region of real space $\mathbb{R}^{2^{n}}$ corresponding to entropies is known to define a convex cone [63]; a complete and explicit description remains unknown. For this reason, one has to work with an outer approximation, known as the Shannon cone $\Gamma_{n}$, defined by the set of linear inequalities given by

$$
\begin{aligned}
H([n] \backslash\{i\}) & \leq H([n]), \\
H(S)+H(S \cup\{i, j\}) & \leq H(S \cup\{i\})+H(S \cup\{j\}), \\
H(\emptyset) & =0,
\end{aligned}
$$

for all $S \subset[n] \backslash\{i, j\}, i \neq j$ and $i, j \in[n]$. These inequalities are known as the elementary inequalities and any inequality that follows from the elementary set is said to be of the Shannon type. The first constraint, given in Eq. (15a), is known as monotonicity and states that the uncertainty about a set of variables should always be larger than or equal to the uncertainty about any subset of it, i.e., nonnegativity of conditional entropy. The second constraint, given in Eq. (15b), is called strong subadditivity and is equivalent to the nonnegativity of the conditional mutual information. That is,

$$
\begin{aligned}
& I\left(X_{i}: X_{j} \mid X_{S}\right) \\
& \quad:=H\left(X_{S \cup i}\right)+H\left(X_{S \cup j}\right)-H\left(X_{S \cup\{i, j\}}\right)-H\left(X_{S}\right) \\
& \quad \geq 0 .
\end{aligned}
$$

The causal relations implied by a given causal structure can be easily integrated in this framework as linear constraints. For instance, the independence of the sources in the causal structure of Fig. 3 implies that $H\left(U_{x}, U_{y}, \Lambda\right)=H\left(U_{x}\right)+$ $H\left(U_{y}\right)+H(\Lambda)$. The subspace of $\mathbb{R}^{2^{n}}$ defined by all such constraints can be denoted as $\Gamma_{c}$. Thus, any entropy vector compatible with a given causal structure should lie in the convex cone $\Gamma_{n}^{c}:=\Gamma_{n} \cap \Gamma_{c}$. Since the sources are not directly observable in the Bell experiment, they need to be traced out from our description, an instance of a quantifier elimination problem that in the entropic case can be performed by a simple Fourier-Motzkin elimination [64].

\section{BOUNDING THE MEASUREMENT DEPENDENCE}

As noted earlier, in order to upper bound the measurement dependence $I(X, Y: \Lambda)$, we modify the causal structure to that of Fig. 3, wherein there is an extra variable $R$ that might depend on the sources $U_{x}$ and $U_{y}$ but is independent of $\Lambda$.

Employing the general entropic framework introduced in Ref. [58] and outlined above, we can completely characterize the Shannon inequalities bounding the measurement dependence $I(X, Y: \Lambda)$. For our purpose, the causal constraints implied by the DAG in Fig. 3 can be summarized by the entropic constraints

$$
H\left(U_{x}, U_{y}, \Lambda\right)=H\left(U_{x}\right)+H\left(U_{y}\right)+H(\Lambda),
$$

and

$$
\begin{aligned}
& I\left(R: X, Y, \Lambda \mid U_{x}, U_{y}\right)=0, \\
& I\left(X: R, Y, U_{y} \mid U_{x}, \Lambda\right)=0, \\
& I\left(Y: R, X, U_{x} \mid U_{y}, \Lambda\right)=0,
\end{aligned}
$$

Equation (16a) follows from the independence of the sources, while Eq. (16b) encodes the zero conditional mutual information between any random variable and its causal nondescendants given its parents, i.e., the local Markov condition.

Using the approach delineated before and performing the corresponding Fourier-Motzkin elimination [64], we find three nontrivial upper bounds for $I(X, Y: \Lambda)$. 
Lemma 1: For any data compatible with the classical causal structure in Fig. 3, we find that $I(X, Y: \Lambda) \leq \Theta(X, Y, R)$, where

$$
\Theta(X, Y, R):=\min \left\{\begin{array}{l}
H(X, Y \mid R), \\
H(X, Y)-I(X: Y: R)-I(X: R)-I(Y: R), \\
H(X, Y)+H(R)-2 I(X: Y: R)-2 I(X: R)-2 I(Y: R),
\end{array}\right.
$$

and where the term $I(X: Y: R)$ is the tripartite mutual information, which can be rewritten as $I(X: Y: R):=$ $H(X, Y, R)-H(X, Y)-H(X, R)-H(Y, R)+H(X)+H(Y)+H(R)$.

The entropic approach also gives rise to the lower bound given by

$$
I(X: Y) \leq I(X, Y: \Lambda) .
$$

Each of the upper bounds in Lemma 1 can be combined with Eq. (11) or Eq. (12) to give rise to a nonlinear Bell inequality.

Proposition 2: For observational data compatible with the classical causal structure in Fig. 3, we find that

$$
2-h\left(\frac{4-\mathrm{CHSH}}{8}\right)-\frac{4+\mathrm{CHSH}}{8} \log _{2} 3 \leq \Theta(X, Y, R),
$$

by virtue of combining Eq. (12) with Lemma 1, as well as

$$
\frac{\mathrm{CHSH}-2}{4} \leq \sqrt{\frac{\Theta(X, Y, R)}{\log _{2} e}},
$$

by virtue of combining Eq. (11) with Lemma 1 through the Pinsker inequality given in Eq. (9).

Under the assumption that the sources $U_{x}, U_{y}$, and $\Lambda$ are independent, a violation of any combination of these inequalities would mean that whatever degree of measurement dependence is present, i.e., whatever value $I(X, Y$ : $\Lambda)$ takes, it is not enough to explain the observed correlations. Thus, we would be unambiguously witnessing nonclassicality. Note that if the input variables $X$ and $Y$ are perfectly correlated with the auxiliary variable $R$, then $H(X, Y \mid R)=0$, implying that $I(X, Y: \Lambda)=0$. In this case, we recover the usual Bell scenario with no measurement dependence. It is important to highlight, however, that in our scenario, an upper bound on the amount of measurement dependence is implied by the empirical data (assuming the independence of sources) and not assumed a priori, as in a standard Bell scenario.

Note that the upper bounds in Lemma 1 are valid for an arbitrary number of inputs and outputs. Thus, inequalities such as Eqs. (19) and (20) can be derived for arbitrary bipartite Bell scenarios. To illustrate, in Ref. [17] it has been noted that the measure $\mathcal{M}$ can also be related to the Collins-Gisin-Linden-Massar-Popescu (CGLMP) inequality [65], a Bell inequality bounding classical correlations in a scenario where Alice and Bob have $d$ possible outcomes. More precisely,

$$
\left(I_{d}-2\right) / 4 \leq \mathcal{M},
$$

where the CGLMP inequality is given by [65]

$$
\begin{aligned}
I_{d}:= & \sum_{k=0}^{\lceil d / 2\rceil-1}\left(1-\frac{2 k}{d-1}\right) \\
& \times\left[p\left(a_{0}=b_{0}+k\right)+p\left(b_{0}=a_{1}+k+1\right)\right. \\
& +p\left(a_{1}=b_{1}+k\right)+p\left(b_{1}=a_{0}+k\right) \\
& -p\left(a_{0}=b_{0}-k-1\right)-p\left(b_{0}=a_{1}-k\right) \\
& \left.-p\left(a_{1}=b_{1}-k-1\right)-p\left(b_{1}=a_{0}-k-1\right)\right] \leq 2
\end{aligned}
$$

and where

$$
p\left(a_{x}=b_{y}+k\right)=\sum_{j=0}^{d-1} p\left(a_{x}=j, b_{y}=j+k \bmod d\right),
$$

with $p\left(a_{x}, b_{y}\right)=p(a, b \mid x, y)$. Using the Pinsker inequality given in Eq. (9), we can readily derive the generalization of inequality given in Eq. (20).

Proposition 3: For observational data compatible with the classical causal structure in Fig. 3, we find that

$$
\frac{I_{d}-2}{4} \leq \sqrt{\frac{\Theta(X, Y, R)}{\log _{2} e}} .
$$

Violation of the inequality given in Eq. (24) implies that the degree of violation of the CGLMP inequality cannot be accounted for by measurement dependence and therefore attests to the presence of nonclassicality. 


\section{A. Example: The Fritz distribution}

To illustrate our results, we consider the Fritz distribution [41], the first known example connecting causal networks with Bell's theorem. In this case, all variables are binary and the measurement outcome of variable $r$ consists of two bits, $r=\left(r_{0}, r_{1}\right)$. As argued by Fritz, if the bit $x$ is perfectly correlated with $r_{0}$, this implies that $x$ should be completely uncorrelated from the source $\lambda$. Similarly, perfect correlation between $y$ and $r_{1}$ implies that $y$ is uncorrelated from the source $\lambda$. That is, the variables $X$ and $Y$ can be seen as the standard measurement choices of Alice and Bob in the usual Bell scenario. Under this condition of perfect correlations, the violation of a standard Bell inequality by the conditional distribution $p(a, b \mid x, y)$ is then a sufficient condition to witness the nonclassicality.

A quantum realization of such scenario is given by

$$
\begin{aligned}
& p\left(a, x, b, y, r_{0}, r_{1}\right) \\
& \quad=\operatorname{Tr}\left(\rho_{A B} \otimes \rho_{X R_{0}} \otimes \rho_{Y R_{1}} \cdot M_{a, x}^{A X} \otimes M_{b, y}^{B Y} \otimes M_{r_{0}, r_{1}}^{R_{0} R_{1}}\right),
\end{aligned}
$$

where $\rho_{A B}$ denotes the density operator of the state shared between Alice and Bob (thus replacing the classical description in terms of the hidden variable $\Lambda$ ), and similarly for $\rho_{X R_{0}}$ and $\rho_{Y R_{1}} ;\left\{M_{a, x}^{A X}\right\}$ denotes a positive operator valued measure (POVM) acting on the physical system in Alice's possession (similarly for $\left\{M_{b, y}^{B Y}\right\}$ and $\left\{M_{r_{0}, r_{1}}^{R_{0} R_{1}}\right\}$ ). In the Fritz case, the sources $\rho_{A B}, \rho_{X R_{0}}, \rho_{Y R_{1}}$ are given by three singlet states $|\Phi\rangle=(1 / \sqrt{2})(|00\rangle+|11\rangle)$ and the POVMs have the following form:

$$
\begin{gathered}
M_{\left(r_{0}, r_{1}\right)}^{R_{0} R_{1}}=M_{r_{0}}^{R_{0}} \otimes M_{r_{1}}^{R_{1}}, \\
M_{(a, x)}^{A X}=M_{x}^{X} \otimes M_{a \mid x}^{A}, \\
M_{(b, y)}^{B Y}=M_{y}^{y} \otimes M_{b \mid y}^{B},
\end{gathered}
$$

where $\left\{M_{r_{0}}^{R_{0}}\right\},\left\{M_{r_{1}}^{R_{1}}\right\},\left\{M_{x}^{X}\right\},\left\{M_{y}^{Y}\right\}$ are all measurements of the $\sigma_{z}$ basis, $\left\{M_{a \mid x}^{A}\right\}$ corresponds to one of the two Pauli observables among $\left\{\sigma_{x}, \sigma_{z}\right\}$ depending on the value of $x$, and $\left\{M_{b \mid y}^{B}\right\}$ corresponds to one of the two observables among $\left\{\left(\sigma_{z}+\sigma_{x}\right) / \sqrt{2},\left(\sigma_{z}-\sigma_{x}\right) / \sqrt{2}\right\}$ depending on the value of $y$. The fact that the measurements in Fritz's example have been chosen to ensure that the conditional $p(a, b \mid x, y)$ violates a Bell inequality implies that Fritz's distribution $p\left(a, x, b, y, r_{0}, r_{1}\right)$ has no classical explanation.

Any experiment that aims to realize the Fritz distribution in the triangle scenario aims to realize the ideal states and measurements specified above but due to the inevitability of noise, the states and measurements that are actually implemented are necessarily noisy versions of these. Consider, for instance, that the source states are noisy versions of the Bell state, given by $\rho=v|\Phi\rangle\langle\Phi|+(1-v) \mathbb{1} / 4$.
This implies that the correlations between $x$ and $r_{0}$ and between $y$ and $r_{1}$ are not perfect and that the Fritz argument can no longer be employed. Even though in this case we do not have any measurement dependence, the point is that the correlations generated by such a model are indistinguishable from a measurement-dependent model. In other terms, to be sure about the nonclassicality of the data, we have to employ the causal network delineated above. For this case, however, $\Theta(X, Y, R)$ is given by

$$
2-\frac{s\left((v-1)^{2}\right)+s\left((v+1)^{2}\right)+s\left(1-v^{2}\right)}{4},
$$

where $s(x):=x \log _{2}(x)$, implying that visibilities as high as $v \approx 0.994$ are required to observe any violation of the inequalities given in Eqs. (9) or (12) and thus witness nonclassicality even in the potential presence of measurement dependence. It is worth pointing out, however, that the source shared between $X$ and $R_{0}$ and the source between $Y$ and $R_{1}$ do not need to have a quantum nature. Since we are simply measuring such states in the computational basis, the same correlations can be achieved with a classical source, significantly simplifying an experimental test.

In hindsight, it is not surprising that the inequalities we derive are not robust. It is known that measurement dependence is a very strong resource to simulate nonlocal correlations in a Bell scenario [10,20]. Remarkably, however, different approaches that do not hinge on Bell's theorem can tolerate a significant amount of measurement dependence, way beyond what is possible within the standard Bell scenario. As will be explored in detail elsewhere, resorting to the inflation technique using "Web" inflation [59, Fig. 2] we can derive new nonlinear inequalities allowing for visibilities as low as $v \approx 0.907$; indeed, showing that the causal network we propose here not only leads to testable constraints but can also tolerate much more measurement dependence than usual approaches.

\section{B. Multipartite Bell inequalities without measurement independence}

So far, we have focused on the bipartite scenario but our results can be readily extended beyond this. Concretely, consider the case of $n$ parties, each $i$ th part with an input $X_{i}$ and output $A_{i}$; the cardinalities of the inputs and outputs being arbitrary (see Fig. 4).

Similarly to the bipartite case, we introduce an auxiliary variable $R$ that can depend on all the sources of locallaboratory private randomness $\left\{U_{i}\right\}_{i}$, where $U_{i}$ accounts for causal influences on $X_{i}$ and $A_{i}$ that are independent of $\Lambda$ (with $i=1, \ldots, n$ ) (see Fig. 5). The joint distributions over the observed variables that are compatible with the causal 


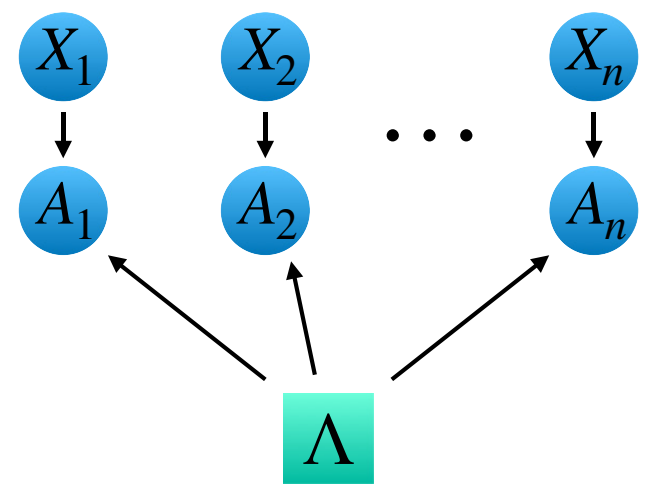

FIG. 4. The causal structure of a standard multipartite Bell scenario. Each of the $n$ distant parties has a common source $\Lambda$ and inputs and outputs labeled as $X_{i}$ and $A_{i}$, respectively.

structure of Fig. 5 are

$$
\begin{aligned}
p & \left(a_{1}, \ldots, a_{n}, x_{1}, \ldots x_{n}, r\right) \\
\quad & =\sum_{u_{1}, \ldots, u_{n}, \lambda} p\left(r \mid u_{1}, \ldots, u_{n}\right) p(\lambda) \prod_{i=1}^{n} p\left(a_{i}, x_{i} \mid u_{i}, \lambda\right) p\left(u_{i}\right) .
\end{aligned}
$$

Observational statistics over the original observable variables together with $R$ can then be employed to upper bound $I\left(X_{1}, \ldots, X_{n}: \Lambda\right)$.

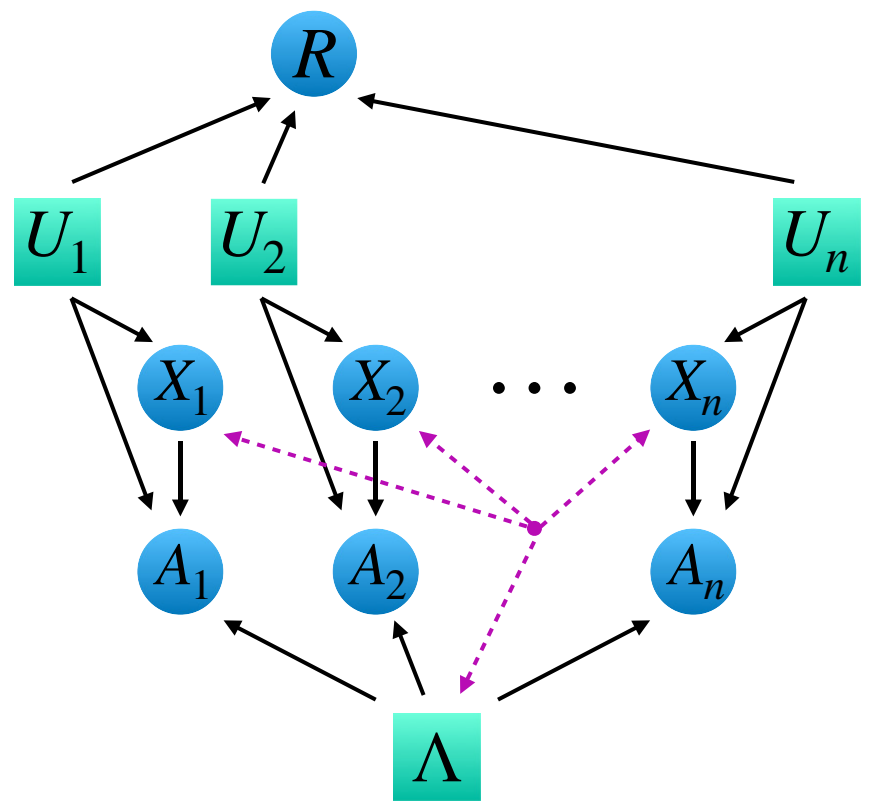

FIG. 5. A causal structure assessing measurement dependence in a multipartite Bell scenario. The auxiliary variable $R$ allows one to practically upper bound the amount of measurement dependence that might be present. The double-arrowed (purple) edges indicate that the correlations between the input variables $X_{i}$ and $\Lambda$ might arise from direct causal influence or via a common source.
Lemma 4: For any data compatible with the classical causal structure in Fig. 5, we find that

$$
I\left(X_{1}, \ldots, X_{n}: \Lambda\right) \leq H\left(X_{1}, \ldots, X_{n} \mid R\right) .
$$

Proof. To prove Lemma 4, we collect all Bell-scenario inputs into the composite random variable $\boldsymbol{X}=\left(X_{1}, \ldots, X_{n}\right)$ and all Bell-scenario outputs into the composite random variable $\boldsymbol{A}=\left(A_{1}, \ldots, A_{n}\right)$. We then combine the Shannontype inequality

$$
H(R, \Lambda)+H(\boldsymbol{X})-H(\boldsymbol{X}, \Lambda) \leq H(\boldsymbol{X} \mid R)+H(R)
$$

with the minimal causal assumption that $R$ is independent of $\Lambda$ such that

$$
H(R, \Lambda)=H(R)+H(\Lambda)
$$

to obtain Lemma 4 via the substitution $H(\Lambda)+H(\boldsymbol{X})-$ $H(\boldsymbol{X}, \Lambda)=I(\boldsymbol{X}: \Lambda)$.

Suppose that we have a generic result that relates the violation of some Bell-type function $I$ to the L1-norm quantifier $\mathcal{M}$, i.e.,

$$
\begin{aligned}
f(I) & \leq \mathcal{M}, \quad \text { where } \\
\mathcal{M} & :=\sum_{x_{1} \ldots x_{n}, \lambda}\left|p\left(x_{1}, \ldots, x_{n}, \lambda\right)-p\left(x_{1}, \ldots, x_{n}\right) p(\lambda)\right|,
\end{aligned}
$$

and where $f$ is some linear function for which $f(I) \leq 0$ for any correlations not violating the Bell inequality [with $f(I)>0$ otherwise]. Combining Eq. (32) with Lemma 4 via the Pinsker inequality given in Eq. (9), we then generically obtain

$$
f(I) \leq \sqrt{\frac{H\left(X_{1}, \ldots, X_{n} \mid R\right)}{\log _{2} e}} .
$$

Clearly, if $H\left(X_{1}, \ldots, X_{n} \mid R\right)=0$, we recover the usual measurement-independent case characterized by the Bell inequality $f(I) \leq 0$.

Instead of using the Pinsker inequality to connect the L1-norm quantifier $\mathcal{M}$ with the information-theoretical measure $I\left(X_{1}, \ldots, X_{n}: \Lambda\right)$, we can try to derive lower bounds for the latter by exploring multipartite inequalities.

This is how we achieve the following proposition, for the tripartite Bell scenario, by adapting the results in [10] to the Mermin-Ardehali-Belinski-Klyshko [38,66,67] inequality

$$
\begin{aligned}
M & :=\left\langle A_{0} B_{0} C_{1}\right\rangle+\left\langle A_{0} B_{1} C_{0}\right\rangle+\left\langle A_{1} B_{0} C_{0}\right\rangle-\left\langle A_{1} B_{1} C_{1}\right\rangle \\
& \leq 2 .
\end{aligned}
$$


Proposition 5: For observational data compatible with the classical causal structure in Fig. 5 specialized to the case of three parties, we find that

$$
1-\frac{1}{2} h\left(\frac{4-M}{8}\right)-\frac{4+M}{16} \log _{2} 3 \leq H(X, Y, Z \mid R)
$$

whenever the distribution over the inputs is uniform, i.e., when $p(x, y, z)=1 / 8$.

Proof. As we show in Appendix A, assuming $p(x, y, z)=$ $1 / 8$, we obtain

$$
1-\frac{1}{2} h\left(\frac{4-M}{8}\right)-\frac{4+M}{16} \log _{2} 3 \leq I(X, Y, Z: \Lambda),
$$

which is the same for signaling and no-signaling behaviors (see Appendix B). We then obtain Proposition 5 by combining Eq. (36) with Lemma 4.

It is not straightforward to compare the lower bound on the amount of measurement dependence required to explain a given degree of violation in the case of the Mermin inequality [Eq. (36)] versus the case of the CHSH inequality [Eq. (12)], as the Mermin inequality can be violated quantumly up to its algebraic maximum, while the CHSH inequality cannot. We therefore consider the degree of measurement dependence as a function of the

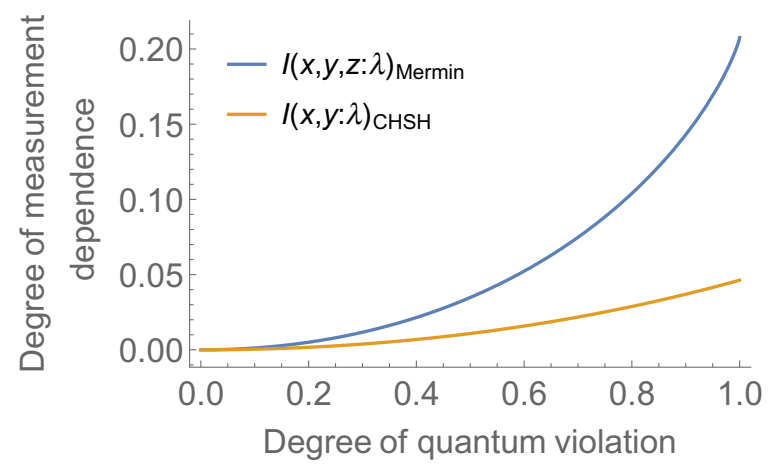

FIG. 6. Measurement dependence and Bell-inequality violations. The orange curve shows the lower bound on the measurement dependence $I(X, Y: \Lambda)$ as described by Eq. (12), needed to explain a given degree of quantum violation for the $\mathrm{CHSH}$ inequality given by $(\mathrm{CHSH}-2) / 2 \sqrt{2}$. The blue curve shows the lower bound on the measurement dependence $I(X, Y, Z: \Lambda)$ as described by Eq. (36), needed to explain a given degree of quantum violation for the Mermin inequality given by $(M-2) / 4$. A comparison between the two curves shows that a higher degree of measurement dependence is required to explain the violation of the Mermin inequality with the same degree of quantum violation as the $\mathrm{CHSH}$ case, a point that can be of experimental relevance when trying to violate measurement-dependent Bell inequalities. ratio between the violation and the maximum quantum violation. The result is plotted in Fig. 6, which demonstrates that Mermin requires more measurement dependence to explain away comparable violation ratios than $\mathrm{CHSH}$.

Moreover, by assuming that some inputs never happen (as it is the case in the Mermin inequality), one obtains a lower bound for $I(X, Y, Z: \Lambda)$ that is exactly the same as in the CHSH scenario (see Appendix A), indicating that it is possible to explore biased distribution of inputs in the analysis of measurement dependence.

\section{RELATING CAUSAL NETWORKS TO RELAXATIONS OF MEASUREMENT INDEPENDENCE}

So far, we have restricted our attention to the analysis of measurement dependence in standard Bell scenarios where the correlations between the distant parties is mediated by a single source. More recently, a number of new causal scenarios have started to be considered, typically consisting of many independent sources [39-47,49]. The paradigmatic example is the so-called triangle scenario, shown in Fig. 7. Its most prominent feature is that it can lead to nonlocal correlations even though it has no input variables [41,49], an ingredient that until then has been considered essential for the appearance of nonclassical behavior.

In spite of the growing theoretical and experimental attention [36], progress in the analysis of nonclassical behavior in such causal structures has been hampered by the fact that the set of correlations they define is nonconvex and very difficult to characterize $[44,59]$. In the following, we show how our results for Bell scenarios with measurement dependence can be readily applied to derive new nonlinear Bell inequalities for different classes of causal networks.

For the sake of an example, we start by focusing on the triangle network. The most general correlations admissible

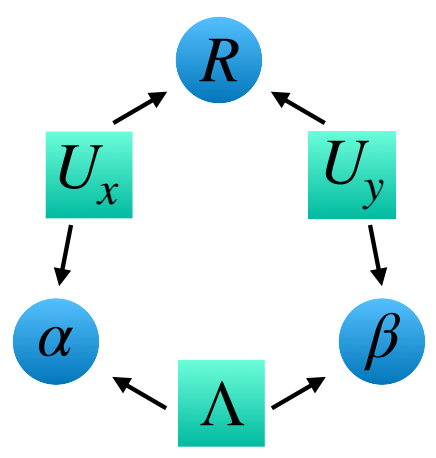

FIG. 7. The triangle network. Pairwise-independent sources generate the correlations between the three observable variables $\alpha, \beta$, and $R$. 
in the triangle network have the form

$$
\begin{aligned}
p(\alpha, \beta, r)= & \sum_{u_{x}, u_{y}, \lambda} p\left(\alpha \mid u_{x}, \lambda\right) p\left(\beta \mid u_{y}, \lambda\right) p\left(r \mid u_{x}, u_{y}\right) \\
& \times p\left(u_{x}\right) p\left(u_{y}\right) p(\lambda)
\end{aligned}
$$

which is precisely the same form as Eq. (14) under the association $\alpha \leftrightarrow(A, X)$ and $\beta \leftrightarrow(B, Y)$.

As shown in Ref. [41], standard Bell-scenario correlations can be mapped onto the triangle network, thus providing the first proof that such a causal structure can support nonclassical correlations. We generalize this result by noting that correlations in the nonstandard Bell scenario with measurement dependence of Fig. 3 can be mapped bijectively onto the triangle network via the common forms of Eqs. (14) and (37). This two-way mapping allows us to translate results both ways between those scenarios.

Note that we relax the assumption that $X$ has a direct causal influence over $A$. Upon allowing for measurement dependence, there is no further loss of generality in treating $A$ and $X$ on an equal footing, i.e., as a single composite outcome variable $\alpha=(A, X)$ that is a function of the sources $U_{x}$ and $\Lambda$ [68]. We similarly merge $B$ and $Y$ into the single composite variable $\beta=(B, Y)$. As detailed in the proof of Lemma 4, the bounds on the measurement dependence $I(X, Y: \Lambda)$ only assume such general dependence. Thus, all the results we derive above can be directly applied to the triangle network.

Lemma 6: Let $\mathcal{G}_{\text {Bell-MI+aux }}$ be the bipartite Bell scenario without the assumption of measurement independence supplemented with an auxiliary variable $R$ as per Fig. 3. Let $\mathcal{G}_{\text {triangle }}$ be the causal scenario depicted in Fig. 7. Then, a distribution $P(a, b, x, y, r)$ is incompatible with $\mathcal{G}_{\text {Bell-MI+aux }}$ if and only if $P(\alpha=(a, x), \beta=(b, y), r)$ is incompatible with $\mathcal{G}_{\text {triangle. }}$

Corollary 6.1: Any correlations compatible with the classical triangle network should respect the nonlinear inequalities given in Eqs. (19), (20), and (24).

Corollary 6.2: For the special case of triangle-scenario correlations where $H(X, Y \mid R)=0$, it follows that if $P(a, b \mid x, y)$ violates a traditional Bell inequality, then $P(\alpha=(a, x), \beta=(b, y), r)$ is incompatible with $\mathcal{G}_{\text {triangle }}$.

As a consequence of Lemma 6, our results generalize the result of Fritz [41] in a number of ways, since Fritz's original argument given there was only applicable when $H(X, Y \mid R)=0$ and, furthermore, was unable to explicitly derive a testable Bell inequality. It is worth pointing out that different Bell inequalities able to witness quantum nonlocality in the triangle have already been derived [49,69,70]. In particular, in Ref. [69], a specific inequality has been obtained for testing the mapping between a standard Bell scenario and the triangle network as proposed in Ref. [41]. Similarly, our inequalities given in Eqs. (19) and (20) also witness the nonclassical behavior whenever the CHSH inequality is violated [if $H(X, Y \mid R)=0]$.

Moreover, our construction can be extended to prove the possibility of nonclassical behavior in causal networks of growing size and where the variables can assume different cardinalities. As a generalization of the triangle causal structure, we consider any causal network inspired by the so-called bipartite graphs [71,72] and composed of two layers: a first layer corresponding to $o+s$ sources labeled as $\left\{\Lambda_{1}, \ldots, \Lambda_{o}, U_{1}, \ldots, U_{s}\right\}$ acting as common causes to the $n+m$ observable variables $\alpha_{1}, \ldots, \alpha_{n}, R_{1}, \ldots, R_{m}$. In the following, we restrict our attention to two particular classes of these general networks and, in particular, derive nonlinear Bell inequalities that can be violated by quantum correlations in those networks. In the first class, we fix $o=1$ and let $s=n$, whereas in the second class, we fix $s=2$ and let $o=n-2$.

\section{A. "2's \& n" networks}

As a first case, we consider networks of $n+1$ parties in which all parties except for $R$ are connected by an $n$-way source, whereas $R$ is connected to every other individual party by a 2-way source. An example of this is given in Fig. 8. We call such a scenario a " 2 's \& $n$ " network. As a second case, we consider the cyclic network of degree $n$, where $n-2$ sources $\Lambda_{i}$ connect pairs of $\alpha_{i}$ 's (with $i=1, \ldots, n)$, source $U_{1}$ connects $\alpha_{1}$ to $R$, and $U_{2}$ connects $\alpha_{n}$ to $R$ (see Fig. 9). A "2's $\& n$ " network admits

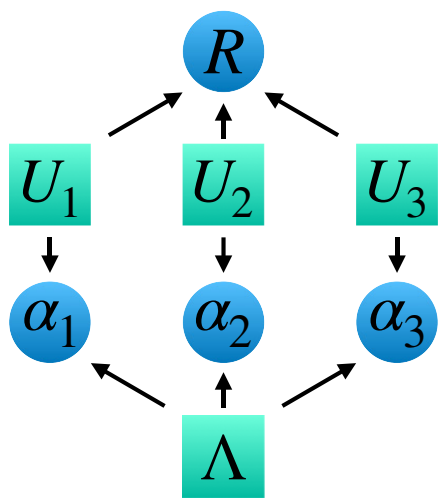

FIG. 8. The "2's \& $\boldsymbol{n}$ " network for $n=3$. One observable source connects three of the four observable variables; however, the other sources only connect pairs. More generally, a " 2 's \& $n$ " consists of observable variables $\alpha_{1}, \ldots, \alpha_{n}$ in addition to $R$. Note that the triangle network (see Fig. 7) is a " 2 's \& $n$ " network with $n=2$. 


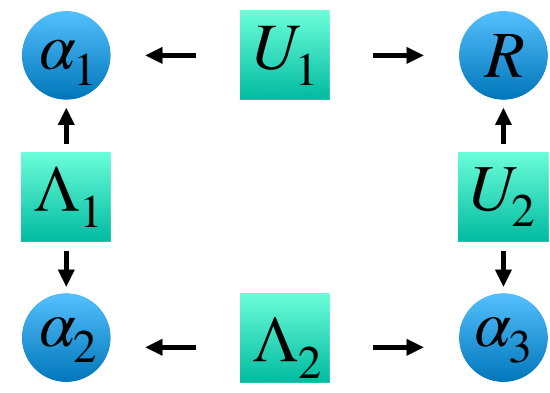

FIG. 9. A cyclic causal structure with $n=4$. Each observable variable its connected to its neighbor via a common source. Note that the triangle network (see Fig. 7) is a particular case of a cyclic network with $n=3$. We index the observable variables in an $n$-cyclic scenario as $\alpha_{1}, \ldots, \alpha_{n-1}$ along with $R$.

distributions of the form

$$
\begin{aligned}
p & \left(\alpha_{1}, \ldots, \alpha_{n}, r\right) \\
& =\sum_{u_{1}, \ldots, u_{n}, \lambda} p\left(r \mid u_{1}, \ldots, u_{n}\right) p(\lambda) \prod_{i=1}^{n} p\left(\alpha_{i} \mid u_{i}, \lambda\right) p\left(u_{i}\right) .
\end{aligned}
$$

The mapping between "2's \& $n$ " networks and multipartite Bell scenarios with measurement dependence and an auxiliary variable $R$ is readily evident by comparing the forms of Eqs. (28) and (38). The are equivalent under the relabeling $\alpha_{i} \leftrightarrow\left(A_{i}, X_{i}\right)$.

Lemma 7: Let $\mathcal{G}_{\text {MultiBell-MI+aux }}$ be the multipartite Bell scenario without the assumption of measurement independence supplemented with an auxiliary variable $R$ as per Fig. 5. Let $\mathcal{G}$ "2's\&n" be a causal scenario of the family depicted in Fig. 8. Then, a distribution $P\left(a_{1}, \ldots, a_{n}, x_{1}, \ldots x_{1}, r\right)$ is incompatible with $\mathcal{G}_{\text {MultiBell-MI+aux }}$ if and only if $P\left[\alpha_{1}=\left(a_{1}, x_{1}\right), \ldots, \alpha_{n}=\right.$ $\left.\left(a_{n}, x_{n}\right), r\right]$ is incompatible with $\mathcal{G}$ " $2^{\prime} s \& n$ ".

Corollary 7.1: Any correlations compatible with the "2's $\& n$ " network for $n=3$ depicted in Fig. 8 should respect the nonlinear inequality given in Eq. (35).

Corollary 7.2: For the special case of " 2 's \& $n$ " scenario correlations where $H\left(X_{1}, \ldots, X_{n}, \mid R\right)=0$, it follows that if $P\left(a_{1}, \ldots, a_{n} \mid x_{1}, \ldots, x_{n}\right)$ violates a traditional multipartite Bell inequality, then $P\left[\alpha_{1}=\left(a_{1}, x_{1}\right), \ldots, \alpha_{n}=\left(a_{n}, x_{n}\right), r\right]$ is incompatible with $\mathcal{G}$ " 2 's\&n".

First, note that the generic multipartite bounds implied by combining the inequality given in Eq. (32) with Lemma 4 remain valid for " 2 's \& $n$ " networks, thus providing a general nonlinear Bell inequality of the form (33) for it. To see that, note that in the proof of Lemma 4, the crucial step given in Eq. (31), which invokes the causal structure under analysis, only makes use of the causal assumption that $R$ is independent of $\Lambda$, a condition fulfilled by " 2 's $\& n$ " networks. As a consequence, the generic nonlinear Bell inequality given in Eq. (32) holds, where $I$ is a function of the conditional probability distribution $p\left(a_{1}, \ldots, a_{n} \mid x_{1}, \ldots, x_{n}\right)$.

\section{B. Cyclic networks}

Next, we consider the cyclic network. By mapping it onto an $n$-locality scenario [39,40,73], we are able to solve an open problem in the characterization of quantum correlations in networks. More specifically, although it has been proven in Ref. [41] that the cyclic scenario of Fig. 9 gives rise to nonclassical correlations, the proof there relies on nonclassicality of a postquantum nature [74]. It has been left open whether a nonclassical behavior that is quantumly realizable is possible. The basic idea is to map the bilocality scenario shown in Fig. 10 onto this cyclic scenario with $n=4$. Note that, in this particular bilocality scenario, there are no external inputs acting as causal parents of the central node $A_{2}$. Thus we achieve a mapping by setting $\alpha_{1}=\left(A_{1}, X_{1}\right), \alpha_{2}=A_{2}$, and $\alpha_{3}=\left(A_{3}, A_{3}\right)$.

There are many nonlinear Bell inequalities $I \leq L$ [where $I$ is a polynomial function of $\left.p\left(a_{1}, a_{2}, a_{3} \mid x_{1}, x_{3}\right)\right]$ derived to characterize the causal structure of the bilocality scenario and that can be violated by quantum correlations. As an example, we have the bilocality inequality $[39,40]$

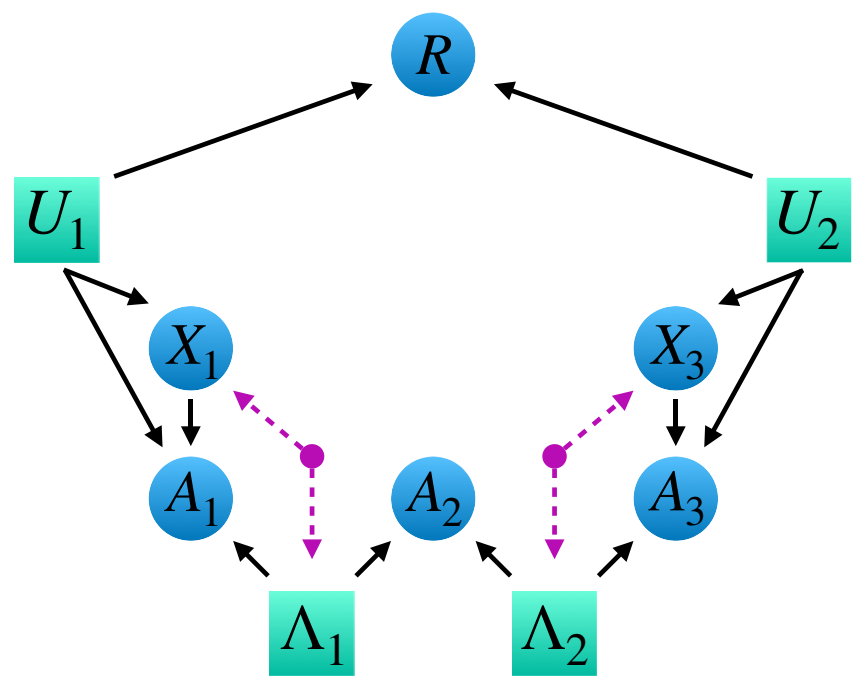

FIG. 10. A bilocality causal structure, shown here with measurement dependence (sources and local inputs potentially correlated) along with an auxiliary variable $R$. In the absence of measurement dependence, this scenario is akin to an entanglementswapping scenario [75], with a central node sharing correlations with two peripheral nodes via two independent sources. More generally, in an $n$-locality scenario with measurement dependence and an auxiliary variable, we have a chain of outcome variables $A_{1}, \ldots, A_{n+1}$ along with $R$ and only the two peripheral nodes $A_{1}$ and $A_{n+1}$ have inputs. 


$$
\begin{aligned}
& \sqrt{I}+\sqrt{J} \leq 2, \quad \text { where } \\
& I:=\sum_{x_{1}, x_{3}}\left\langle a_{1}^{x_{1}} a_{2} a_{3}^{x_{3}}\right\rangle, \quad \text { and } \\
& J:=\sum_{x_{1}, x_{3}}(-1)^{x_{1}+x_{3}}\left\langle a_{1}^{x_{1}} a_{2} a_{3}^{x_{3}}\right\rangle, \quad \text { and } \\
& \left\langle a_{1}^{x_{1}} a_{2} a_{3}^{x_{3}}\right\rangle:=\sum_{a_{1}, a_{2}, a_{3}}(-1)^{a_{1}+a_{2}+a_{3}} p\left(a_{1}, a_{2}, a_{3} \mid x_{1}, x_{3}\right),
\end{aligned}
$$

and where all input and output variables assume the values 0 or 1.

Lemma 8: Let $\mathcal{G}_{n \text {-locality-MI+aux }}$ be the n-locality scenario without the assumption of measurement independence supplemented with an auxiliary variable $R$ as per Fig. 10 and generalizations thereof. Let $\mathcal{G}_{(n+2)-c y c l i c}$ be the causal scenario depicted in Fig. 9 and generalizations therefore. Then, a distribution $P\left(a_{1}, a_{2}, \ldots a_{n}, a_{n+1}, x_{1}, x_{n+1}, r\right)$ is incompatible with $\mathcal{G}_{n-l o c a l i t y-M I+a u x}$ if and only if $\left[\alpha_{1}=\left(a_{1}, x_{1}\right), \alpha_{2}=a_{2}, \ldots, \alpha_{n+1}=\left(a_{n+1}, x_{n+1}\right), r\right]$ is incompatible with $\mathcal{G}_{(n+2)-\text { cyclic }}$.

Corollary 8.1: For the special case of n-cyclic scenario correlations where $H\left(X_{1}, X_{n-1} \mid R\right)=0$, it follows that if $P\left(a_{1}, a_{2}, \ldots, a_{n-1} \mid x_{1}, x_{n-1}\right)$ violates a traditional $n$-locality inequality, then $P\left[\alpha_{1}=\left(a_{1}, x_{1}\right), \alpha_{2}=a_{2}, \ldots, \alpha_{n-1}=\left(a_{n+1}\right.\right.$, $\left.\left.x_{n-1}\right), r\right]$ is incompatible with $\mathcal{G}_{\text {cyclic }}$.

Following the recipe in Ref. [17], one can also relate the violation of such inequalities with the degree of measurement dependence $\mathcal{M}:=\sum_{\lambda_{1}, \lambda_{2}} \mid p\left(x_{1}, x_{3}, \lambda_{1}, \lambda_{2}\right)-$ $p\left(x_{1}, x_{3}\right) p\left(\lambda_{1}\right) p\left(\lambda_{2}\right) \mid$ required to explain it. As before, one can then generally write $g(I) \leq \mathcal{M}$, where now $g$ is a polynomial function of the Bell inequality $I$ such that $g(I)>0$ if the inequality is violated and $g(I) \leq 0$ otherwise. Thus, we also obtain

$$
g(I) \leq \sqrt{\frac{H\left(X_{1}, X_{3} \mid R\right)}{\log _{2} e}} .
$$

In particular, if $X_{1}$ and $X_{3}$ are perfectly correlated with and $R$, then $H\left(X_{1}, X_{3} \mid R\right)=0$ and the quantum violation of any bilocality inequality would be enough to witness quantum nonclassicality in such a cyclic network.

Leveraging the linear-chain $n$-locality scenario considered in Ref. [73], one can demonstrate nonclassicality in cyclic networks of arbitrary size. In the linear-chain $n$ locality scenario, we have $n+1$ observable variables $A_{i}$, of which only $A_{1}$ and $A_{n+1}$ have inputs, denoted $X_{1}$ and $X_{n+1}$, respectively. If we treat $\alpha_{1}=\left(a_{1}, x_{1}\right)$ and $\alpha_{N}=$ $\left(a_{n+1}, x_{n+1}\right)$ and otherwise $\alpha_{i}=a_{i}$, and we close the cycle by introducing the sources $U_{1}$ and $U_{2}$ that connect $\alpha_{1}$ and $\alpha_{n}$ to the variable $R$, we obtain the $(n+2)$-cycle causal network. The joint distribution on observed variables that are compatible with this network are

$$
\begin{aligned}
p\left(\alpha_{1}, \ldots \alpha_{n+1}, r\right)= & p\left(r \mid u_{1}, u_{2}\right) p\left(\alpha_{1} \mid u_{1} \lambda_{1}\right) p\left(\alpha_{n+1} \mid u_{2}, \lambda_{n}\right) \\
& \times p\left(\lambda_{1}\right) \prod_{i=2}^{n} p\left(\alpha_{i} \mid \lambda_{i-1}, \lambda_{i}\right) p\left(\lambda_{n}\right) .
\end{aligned}
$$

Compatible distributions in the $n$-locality scenario with measurement dependence and an auxiliary variable $R$, that is, generalizations of Fig. 10, are of precisely the same form but with the relabeling

$$
\alpha_{i} \leftrightarrow \begin{cases}\left(A_{i}, X_{i}\right), & \text { if } i=1 \text { or } i=n+1, \\ A_{i}, & \text { if } 1<i<n+1 .\end{cases}
$$

As before, if $X_{1}$ and $X_{n+1}$ are perfectly correlated with $R$, then $H\left(X_{1}, X_{n+1} \mid R\right)=0$ and the violation of any $n$-locality inequality bounding the linear-chain scenario also suffices to demonstrate nonlocality in the cyclic scenario. An example of such inequality has been provided in Ref. [73].

\section{DISCUSSION AND CONCLUSIONS}

If observed statistical correlations are found to violate a Bell inequality, then one can conclude that these correlations cannot be explained by a classical causal model having the causal structure of Fig. 1. The moniker of "Bell nonlocality" has traditionally been assigned to this phenomenon because one can avoid the contradiction through a modification of the causal structure of Fig. 1 that incorporates a (nonlocal) causal influence from the setting on one side to the outcome on the other. It is well known, however, that there are other opportunities for evading the contradiction. In particular, one can modify the causal structure of Fig. 1 by relaxing the assumption that there is no causal influence from $\Lambda$ to the setting variables $X$ or $Y$ and no common cause of $\Lambda$ and $X$ or of $\Lambda$ and $Y$, that is, by relaxing the assumption of measurement independence. We argue that there are two distinct causal mechanisms by which the assumption of measurement independence might fail in a Bell experiment. The first mechanism is a violation of independence between $\Lambda$ and variables that causally determine the settings $X$ and $Y$. If the settings are made to depend causally on cosmic photons [23,25], then this version of the assumption seems especially plausible, since denying it seems to require assuming a superdeterministic world wherein everything is potentially correlated with everything else. The second mechanism is one wherein some systems that mediate the influence of the ultimate causal determinants of the settings (such as cosmic photons) become influenced by $\Lambda$ or by a variable that also influences $\Lambda$. In other words, even though the 
variables that determine the settings may start out independent of $\Lambda$, they might become correlated with it (e.g., when the cosmic photons enter the laboratory). It is this second class of mechanism for measurement dependence that we here show can be subjected to an experimental test.

We embed the Bell causal structure in a larger network and assume the independence of the variables that causally determine the settings (i.e., we rule out, by assumption, the first mechanism for achieving measurement dependence) and show that in this case it is possible to upper bound the amount of measurement dependence (of the second kind) based on observational data. Combining these upper bounds with previous lower bounds for the amount of measurement dependence required to explain a given violation of Bell inequalities, we are able to derive nonlinear Bell-type inequalities the violation of which is a proof of nonclassicality in spite of the presence of some measurement dependence (of the second kind).

To our knowledge, this is the first demonstration of the possibility of putting an upper bound on the amount of measurement dependence in a Bell experiment, a feature that deserves further theoretical and experimental investigation. It is worth remarking that the results in Ref. [70] can be understood as complementary to ours. There, focusing on the triangle network, it has been shown that under the assumption of perfect correlation between some variables (ruling out any possible measurement dependence of the second kind) they can witness nonclassicality even allowing correlations between the sources (thus allowing measurement dependence of the first kind, even though the latter cannot be upper bounded by observational data).

Following that, we also show how measurementdependent Bell causal structures can be readily mapped onto causal networks of growing size and complexity, a field of research that is attracting growing attention but for which advances have been hampered by the difficulty in deriving Bell inequalities. By doing so, we derive a robust Bell inequality especially suited to test the Fritz correlations [41] in the triangle network and which, in contrast to previous attempts, does not require perfect correlations between some subset of variables [70], which is a welcome feature for achieving an experimental implementation. Finally, by mapping fully connected networks onto multipartite Bell scenarios and by mapping cyclic networks to the linear $n$-locality scenario, we are able to show that such networks can give rise to correlations that witness nonclassicality.

We believe that our results are just a first step toward a better understanding of measurement-dependent causal models and how these can be tested experimentally. For instance, upper bounds such as the one in Eq. (33) employ the Pinsker inequality, which is known to be nontight. Can we employ more modern techniques such as those in
Ref. [20], the covariance [71,76] or the inflation technique [59], in order to provide better lower and upper bounds to $\mathcal{M}$ and $I(X, Y: \Lambda)$ and thus improve our inequalities? We prove that this possible for the tripartite scenario by deriving new results for the Mermin inequality.

It is noteworthy that the analysis we carry out here can also be extended to analyze measurement dependence in different causal structures, in particular in the instrumental scenario (which plays a central role in the field of causal inference). As will be further explored in Ref. [77], measurement independence is also a crucial assumption in causal inference, the violation of which has important consequences for the analysis of cause and effect in empirical data $[78,79]$.

Finally, we believe that the techniques we introduce here can offer a way to tackle open questions in the study of networks. As mentioned before, the first example of nonclassical correlations in the triangle network has been provided by a mapping of that network onto the usual Bell network [41]. Other examples of nonclassicality that do not hinge directly on Bell's theorem are known [49]. However, it remains an open question as to how one can prove that the nonclassicality observed in a given network is truly different from that in Bell's theorem. Further exploration of the connection between Bell scenarios with measurement dependence and networks may offer a way to better understand their similarities and their differences. We hope that our results might trigger future research along all of these directions.

\section{ACKNOWLEDGMENTS}

This work was supported by The John Templeton Foundation via Q-CAUSAL Grant No. 61084 and via The Quantum Information Structure of Spacetime (QISS) Project (qiss.fr) Grant Agreement No. 61466 (the opinions expressed in this publication are those of the authors and do not necessarily reflect the views of the John Templeton Foundation), by the Serrapilheira Institute (Grant No. Serra-1708-15763), the Brazilian National Council for Scientific and Technological Development (CNPq) via the National Institute for Science and Technology on Quantum Information (INCT-IQ) and Grants No. 307172/2017-1 and No. 311375/2020-0, the Brazilian agencies MCTIC and MEC, and by MIUR via PRIN 2017 (Progetto di Ricerca di Interesse Nazionale), Project QUSHIP (2017SRNBRK). This research was supported by the Perimeter Institute for Theoretical Physics. Research at the Perimeter Institute is supported in part by the Government of Canada through the Department of Innovation, Science, and Economic Development Canada and by the Province of Ontario through the Ministry of Colleges and Universities. The opinions expressed in this publication are those of the authors and do not necessarily reflect the views of the supporting institutions. 


\section{APPENDIX A: MUTUAL INFORMATION BOUND FOR MERMIN INEQUALITY}

In this appendix, we follow similar steps to those introduced in Ref. [20] and we derive a tight informational lower bound of the measurement dependence demanded for a given violation of Mermin's inequality. To that aim, we first show how any violation of this inequality imposes bounds on the distribution $p(x, y, z \mid \lambda)$. Then, considering those bounds, we minimize the mutual information $I(X, Y, Z: \Lambda)$.

In this scenario, the probability $p(a, b, c \mid x, y, z)$, for $a, b, c, x, y, z \in\{0,1\}$, can be factorized as

$$
p(a, b, c \mid x, y, z)=\sum_{\lambda \in \Lambda} p(\lambda \mid x, y, z) \delta_{a f_{\lambda}(x)} \delta_{b, f_{\lambda}(y)} \delta_{c, f_{\lambda}(z)},
$$

In which case the full correlators are given by

$$
\left\langle A_{x} B_{y} C_{z}\right\rangle=\sum_{\lambda} A_{x}(\lambda) B_{y}(\lambda) C_{z}(\lambda) p(\lambda \mid x, y, z)
$$

for $A_{x}, B_{y}, C_{z} \in\{-1,1\}$, given by

$$
\begin{aligned}
& A_{x}(\lambda)=\sum_{a}(-1)^{a} \delta_{a, f_{\lambda}(x)}, \\
& B_{y}(\lambda)=\sum_{b}(-1)^{b} \delta_{b, f_{\lambda}(y)}, \\
& C_{z}(\lambda)=\sum_{c}(-1)^{c} \delta_{c, f_{\lambda}(z)} .
\end{aligned}
$$

Recall that Mermin's inequality $[38,66,67]$ reads

$$
M=\sum_{(x, y, z) \in O}(-1)^{x y z}\left\langle A_{x} B_{y} C_{z}\right\rangle \leq 2,
$$

in which $O$ denotes the set of odd total parity outcomes, i.e., $O=\{(1,0,0),(0,1,0),(0,0,1),(1,1,1)\}$.

Substituting via Eq. (A2), applying Bayes' rule such that $p(\lambda \mid x y z)=p(x, y, z \mid \lambda) p(\lambda) / p(x, y, z)$, and setting $p(x, y, z)=1 / 8$, we obtain

$M=8 \sum_{\lambda} \sum_{(x, y, z) \in O}(-1)^{x y z} A_{x}(\lambda) B_{y}(\lambda) C_{z}(\lambda) p(x, y, z \mid \lambda) p(\lambda)$.

By defining the sets $\mathcal{L}_{\mu, \eta, \nu}$, for $\mu, \eta, v \in\{0,1\}$, as

$$
\begin{aligned}
& \mathcal{L}_{\mu, \eta, v}=\left\{\lambda \in \Lambda \mid A_{1}(\lambda)=(-1)^{\mu} A_{0}(\lambda),\right. \\
& \left.B_{1}(\lambda)=(-1)^{\eta} B_{0}(\lambda), C_{1}(\lambda)=(-1)^{v} C_{0}(\lambda)\right\},
\end{aligned}
$$

we can rewrite $M$ as follows:

$$
M=8 \sum_{\mu, \eta, \nu} \sum_{\lambda \in \mathcal{L}_{\mu, \eta, \nu}} A_{0}(\lambda) B_{0}(\lambda) C_{0}(\lambda) p(\lambda) \Gamma_{\mu, \eta, \nu},
$$

in which

$$
\Gamma_{\mu, \eta, \nu}=\sum_{(x+y+z) \in O}(-1)^{x y z+\mu x+\eta y+v z} p(x, y, z \mid \lambda) .
$$

Recognizing that $p(O \mid \lambda)=p(0,0,1 \mid \lambda)+p(0,1,0 \mid \lambda)+$ $p(1,0,0 \mid \lambda)+p(1,1,1 \mid \lambda)$, we can write

$$
\Gamma_{\mu, \eta, v}=(-1)^{\mu \eta+\mu \nu+\eta v}[p(O \mid \lambda)-2 p(\eta \oplus v \oplus 1, \mu \oplus v \oplus 1, \mu \oplus \eta \oplus 1 \mid \lambda)]
$$

in which $\oplus$ represents sum $\bmod 2$.

Using the above result in Eq. (A5),

$$
\begin{aligned}
& M=\sum_{\mu, \eta, v} \sum_{\lambda \in \mathcal{L}_{\mu, \eta, v}}(-1)^{\mu \eta+\mu \nu+\eta v} A_{0}(\lambda) B_{0}(\lambda) C_{0}(\lambda) p(\lambda) p(O \mid \lambda) \\
& \quad[1-2 p(x=\eta \oplus v \oplus 1, y=\mu \oplus v \oplus 1, z=\mu \oplus \eta \oplus 1 \mid \lambda, O)] .
\end{aligned}
$$

By choosing $A_{0}(\lambda) B_{0}(\lambda) C_{0}(\lambda)=\operatorname{sgn}\left\{(-1)^{\mu \eta+\mu \nu+\eta v}[1-2 p(x=\eta \oplus v \oplus 1, y=\mu \oplus v \oplus 1, z=\mu \oplus \eta \oplus 1 \mid \lambda, O)]\right\}$, for $\lambda \in \mathcal{L}_{\mu, \eta, v}$, we obtain

$$
M \leq 8 \sum_{\mu, \eta, v} \sum_{\lambda \in \mathcal{L}_{\mu, \eta, v}} p(\lambda) p(O \mid \lambda)|1-2 p(x=\eta \oplus v \oplus 1, y=\mu \oplus v \oplus 1, z=\mu \oplus \eta \oplus 1 \mid \lambda, O)| .
$$


For $(x, y, z) \in O$, let $p_{\min } \leq 1 / 4$ be the infimum of $p(x, y, z \mid \lambda, O)$. Then,

$$
\begin{aligned}
M & \leq 8 \sum_{\mu, \eta, v} \sum_{\lambda \in \mathcal{L}_{\mu, \eta, v}} p(\lambda) p(O \mid \lambda)\left(1-2 p_{\min }\right) \\
& \leq 8\left(1-2 p_{\min }\right) p(O) \\
& \leq 4-8 p_{\min },
\end{aligned}
$$

implying that,

$$
p_{\min } \leq \frac{4-M}{8}
$$

saturation is achieved for

$$
p(x=\eta \oplus v \oplus 1, y=\mu \oplus v \oplus 1, z=\mu \oplus \eta \oplus 1 \mid \lambda, O)=p_{\min } \quad \text { or } \quad 1-p_{\min } .
$$

The mutual information reads

$$
\begin{aligned}
I(X, Y, Z: \Lambda) & =H(X, Y, Z)-\sum_{\mu, \eta, \nu} \sum_{\lambda \in \mathcal{L}_{\mu, \eta, \nu}} p(\lambda) H_{\lambda}(X, Y, Z) \\
& =3-\sum_{\mu, \eta, \nu} \sum_{\lambda \in \mathcal{L}_{\mu, \eta, \nu}} p(\lambda) H_{\lambda}(X, Y, Z)
\end{aligned}
$$

and is minimized by making $H_{\lambda}(X, Y, Z)$ as large as possible, which is achieved with distributions $p(x, y, z \mid \lambda)$ as close to a uniform distribution as possible. Thus,

$$
p(x, y, z \mid \lambda)=p_{\min }=\frac{4-M}{8} .
$$

Note above that we choose $p_{\min }$ instead of $1-p_{\min }$; otherwise, we would end up with a distribution further from the uniform one.

For $\lambda \in \mathcal{L}_{\mu, \eta, \nu}$, this leads to distributions of the form

$$
p(x, y, z \mid \lambda)= \begin{cases}\frac{p_{\min }}{2}, & \text { for }(x, y, z)=(\eta \oplus v \oplus 1, \mu \oplus v \oplus 1, \mu \oplus \eta \oplus 1), \\ \frac{1-p_{\min }}{6}, & \text { for }(x, y, z) \in O \text { and }(x, y, z) \neq(\eta \oplus v \oplus 1, \mu \oplus v \oplus 1, \mu \oplus \eta \oplus 1), \\ \frac{1}{8}, & \text { for }(x, y, z) \notin O,\end{cases}
$$

which can be used for each case of $\mu, \eta, v$ always returning the same value for $H_{\lambda}(X, Y, Z)$, so that the mutual information reads

$$
\begin{aligned}
I(X, Y, Z: \Lambda) & \geq 3+\frac{p_{0}}{2} \log p_{0}-\frac{p_{0}}{2} \log 2+\frac{\left(1-p_{0}\right)}{2} \log \left(1-p_{0}\right)-\frac{\left(1-p_{0}\right)}{2} \log 6-\frac{1}{2} \log 8 \\
& =1+\frac{1}{2} h\left(p_{0}\right)-\frac{\left(1-p_{0}\right)}{2} \log 3 \\
& =1+\frac{1}{2} h\left(\frac{4-M}{8}\right)-\frac{4+M}{16} \log 3 .
\end{aligned}
$$

If now we set $p(x, y, z)=1 / 4$ if $(x+y+z) \in O$, then we obtain

$$
M=4 \sum_{\mu, \eta, v} \sum_{\lambda \in \mathcal{L}_{\mu, \eta, \nu}} A_{0}(\lambda) B_{0}(\lambda) C_{0}(\lambda) p(\lambda) \Gamma_{\mu, \eta, v}^{\prime},
$$


in which

$$
\Gamma_{\mu, \eta, v}^{\prime}=(-1)^{\mu \eta+\mu \nu+\eta v}[1-2 p(\eta \oplus v \oplus 1, \mu \oplus v \oplus 1, \mu \oplus \eta \oplus 1 \mid \lambda)] .
$$

While this new constraint leads to the same bound on $p_{\min }$, shown in Eq. (A7), in this case we must adapt the distribution maximizing $H_{\lambda}(x, y, z)$ to the following:

$$
p(x, y, z \mid \lambda)= \begin{cases}p_{\min }, & \text { for } x=\eta \oplus v \oplus 1, y=\mu \oplus v \oplus 1, z=\mu \oplus \eta \oplus 1, \\ \frac{1-p_{\min }}{3}, & \text { for } x \neq \eta \oplus v \oplus 1, \text { or } y \neq \mu \oplus v \oplus 1, \text { or } z \neq \mu \oplus \eta \oplus 1, \text { and } x+y+z \in O, \\ 0, & \text { for } x+y+z \notin O,\end{cases}
$$

for $\lambda \in \mathcal{L}_{\mu, \eta, \nu}$.

This leads to

$$
\begin{aligned}
I(X, Y, Z: \Lambda) & \geq 2+p_{0} \log p_{0}+\left(1-p_{0}\right) \log \left(1-p_{0}\right)-\left(1-p_{0}\right) \log 3 \\
& \geq 2-h\left(\frac{4-M}{8}\right)-\left(\frac{4+M}{8}\right) \log 3 .
\end{aligned}
$$

\section{APPENDIX B: NO-SIGNALING CONDITION}

As in Ref. [20], we also address the question of imposing the extra constraint of no signaling. The answer is exactly the same: the existence of a signaling distribution leading to an arbitrary value of $M$ implies the existence of a no-signaling distribution featuring the same mutual information between the inputs and the hidden variable and value of $M$.

Proof. Assume that there exists a distribution $p(a, b, c \mid$ $x, y, z)$ as in Eq. (A1), which does not satisfy the nosignaling condition and for which $M[p(a, b, c \mid x, y, z)]=$ $M^{*}$. Now, let us build a distribution $\tilde{p}(a, b, c \mid x, y, z)$ as follows:

$$
\begin{aligned}
\tilde{p}(a, b, c \mid x, y, z)= & 8 \sum_{\lambda^{\prime}, \lambda^{\prime \prime} \in\{0,1\}} \sum_{\lambda \in \Lambda} p\left(\lambda^{\prime}, \lambda^{\prime \prime}, \lambda, x, y, z\right) \\
& \times \delta_{a \oplus \lambda^{\prime}, f_{\lambda}(x)} \delta_{b \oplus \lambda^{\prime} \oplus \lambda^{\prime \prime}, f_{\lambda}(y)} \delta_{c \oplus \lambda^{\prime \prime}, f_{\lambda}(z)},
\end{aligned}
$$

in which $p\left(\lambda^{\prime}, \lambda^{\prime \prime}\right)=1 / 4$ and $p\left(\lambda^{\prime}, \lambda^{\prime \prime}, \lambda, x, y, z\right)=1 / 4 p$ $(\lambda, x, y, z)$, which implies that

$$
\begin{aligned}
\sum_{b, c} \tilde{p}(a, b, c \mid x, y, z) & =\sum_{a, c} \tilde{p}(a, b, c \mid x, y, z) \\
& =\sum_{a, b} \tilde{p}(a, b, c \mid x, y, z)=\frac{1}{2} .
\end{aligned}
$$

Another property of this distribution concerns the full correlations $\left\langle\tilde{A}_{x} \tilde{B}_{y} \tilde{C}_{z}\right\rangle$ :

$$
\left\langle\tilde{A}_{x} \tilde{B}_{y} \tilde{C}_{z}\right\rangle=\left\langle A_{x} B_{y} C_{z}\right\rangle .
$$

From Eq. (B1), we can see that $\tilde{p}(a, b, c \mid x, y, z)$ is a no-signalling probability distribution. From Eq. (B1) we have that $M[\tilde{p}(a, b, c \mid x, y, z)]=M^{*}$ and from the fact that $p\left(\lambda^{\prime}, \lambda^{\prime \prime}, \lambda, x, y, z\right)=p\left(\lambda^{\prime}, \lambda^{\prime \prime}\right) p(\lambda, x, y, z)$, we have that $I\left(X, Y, Z: \Lambda, \Lambda^{\prime}, \Lambda^{\prime \prime}\right)=I(X, Y, Z: \Lambda)$.

[1] J. S. Bell, On the Einstein Podolsky Rosen paradox, Phys. Phys. Fizika 1, 195 (1964).

[2] S. Pironio, V. Scarani, and T. Vidick, Focus on device independent quantum information, New J. Phys 18, 100202 (2016).

[3] H. Reichenbach, The Direction of Time Vol. 65 (University of California Press, Berkeley, 1991).

[4] C. J. Wood and R. W. Spekkens, The lesson of causal discovery algorithms for quantum correlations: Causal explanations of Bell-inequality violations require fine tuning, New J. Phys. 17, 033002 (2015).

[5] H. M. Wiseman and E. G. Cavalcanti, in Quantum [Un] Speakables II (Springer, 2017) p. 119.

[6] C. H. Brans, Bell's theorem does not eliminate fully causal hidden variables, Int. J. Theo. Phys. 27, 219 (1988).

[7] J. S. Bell, A. Shimony, M. A. Horne, and J. F. Clauser, An exchange on local beables, Dialectica, 85 (1985).

[8] J. Kofler, T. Paterek, and I. C. V. Brukner, Experimenter's freedom in Bell's theorem and quantum cryptography, Phys. Rev. A 73, 022104 (2006).

[9] D. E. Koh, M. J. W. Hall, Setiawan, J. E. Pope, C. Marletto, A. Kay, V. Scarani, and A. Ekert, Effects of Reduced Measurement Independence on Bell-Based Randomness Expansion, Phys. Rev. Lett. 109, 160404 (2012).

[10] M. J. Hall, Local Deterministic Model of Singlet State Correlations Based on Relaxing Measurement Independence, Phys. Rev. Lett. 105, 250404 (2010).

[11] J. Barrett and N. Gisin, How Much Measurement Independence Is Needed to Demonstrate Nonlocality, Phys. Rev. Lett. 106, 100406 (2011). 
[12] M. J. Hall, Relaxed Bell inequalities and Kochen-Specker theorems, Phys. Rev. A 84, 022102 (2011).

[13] M. Banik, M. R. Gazi, S. Das, A. Rai, and S. Kunkri, Optimal free will on one side in reproducing the singlet correlation, J. Phys. A 45, 205301 (2012).

[14] R. Colbeck and R. Renner, Free randomness can be amplified, Nat. Phys. 8, 450 (2012).

[15] J. Gallicchio, A. S. Friedman, and D. I. Kaiser, Testing Bell's Inequality with Cosmic Photons Closing the SettingIndependence Loophole, Phys. Rev. Lett. 112, 110405 (2014).

[16] G. Putz, D. Rosset, T. J. Barnea, Y.-C. Liang, and N. Gisin, Arbitrarily Small Amount of Measurement Independence Is Sufficient to Manifest Quantum Nonlocality, Phys. Rev. Lett. 113, 190402 (2014).

[17] R. Chaves, R. Kueng, J. B. Brask, and D. Gross, Unifying Framework for Relaxations of the Causal Assumptions in Bell's Theorem, Phys. Rev. Lett. 114, 140403 (2015).

[18] R. Chaves, D. Cavalcanti, and L. Aolita, Causal hierarchy of multipartite Bell nonlocality, Quantum 1, 23 (2017).

[19] A. S. Friedman, A. H. Guth, M. J. Hall, D. I. Kaiser, and J. Gallicchio, Relaxed Bell inequalities with arbitrary measurement dependence for each observer, Phys. Rev. A 99, 012121 (2019).

[20] M. J. Hall and C. Branciard, Measurement-dependence cost for Bell nonlocality: Causal versus retrocausal models, Phys. Rev. A 102, 052228 (2020).

[21] S. Hossenfelder and T. Palmer, Rethinking superdeterminism, Front. Phys. 8, 139 (2020).

[22] D. Aktas, S. Tanzilli, A. Martin, G. Putz, R. Thew, and N. Gisin, Demonstration of Quantum Nonlocality in the Presence of Measurement Dependence, Phys. Rev. Lett. 114, 220404 (2015).

[23] J. Handsteiner, A. S. Friedman, D. Rauch, J. Gallicchio, B. Liu, H. Hosp, J. Kofler, D. Bricher, M. Fink, and C. Leung et al., Cosmic Bell Test Measurement Settings from Milky Way Stars, Phys. Rev. Lett. 118, 060401 (2017).

[24] BIG Bell Test Collaboration and others, Challenging local realism with human choices, Nature 557, 212 (2018).

[25] D. Rauch, J. Handsteiner, A. Hochrainer, J. Gallicchio, A. S. Friedman, C. Leung, B. Liu, L. Bulla, S. Ecker, and F. Steinlechner et al., Cosmic Bell Test Using Random Measurement Settings from High-Redshift Quasars, Phys. Rev. Lett. 121, 080403 (2018).

[26] T. Maudlin, in PSA: Proceedings of the Biennial Meeting of the Philosophy of Science Association (Philosophy of Science Association, 1992), Vol. 1992, p. 404.

[27] G. Brassard, R. Cleve, and A. Tapp, Cost of Exactly Simulating Quantum Entanglement with Classical Communication, Phys. Rev. Lett. 83, 1874 (1999).

[28] M. Steiner, Towards quantifying non-local information transfer: Finite-bit non-locality, Phys. Lett. A 270, 239 (2000).

[29] B. F. Toner and D. Bacon, Communication Cost of Simulating Bell Correlations, Phys. Rev. Lett. 91, 187904 (2003).

[30] T. Maudlin, Quantum Non-Locality and Relativity: Metaphysical Intimations of Modern Physics (John Wiley \& Sons, Hoboken, New Jersey, United States, 2011).
[31] M. Ringbauer and R. Chaves, Probing the non-classicality of temporal correlations, Quantum 1, 35 (2017).

[32] J. B. Brask and R. Chaves, Bell scenarios with communication, J. Phys. A 50, 094001 (2017).

[33] R. D. Gill, The triangle wave versus the cosine: How classical systems can optimally approximate EPR-B correlations, Entropy 22, 287 (2020).

[34] P. Blasiak, E. M. Pothos, J. M. Yearsley, C. Gallus, and E. Borsuk, Violations of locality and free choice are equivalent resources in Bell experiments, Proc. Nat. Ac. Sci. 118, e2020569118 (2021).

[35] J. F. Clauser, M. A. Horne, A. Shimony, and R. A. Holt, Proposed Experiment to Test Local Hidden-Variable Theories, Phys. Rev. Lett. 23, 880 (1969).

[36] A. Tavakoli, A. Pozas-Kerstjens, M.-X. Luo, and M.-O. Renou, Bell nonlocality in networks, ArXiv:2104.10700, (2021).

[37] M. M. Wolf, D. Perez-Garcia, and C. Fernandez, Measurements Incompatible in Quantum Theory Cannot Be Measured Jointly in Any Other No-Signaling Theory, Phys. Rev. Lett. 103, 230402 (2009).

[38] N. D. Mermin, Extreme Quantum Entanglement in a Superposition of Macroscopically Distinct States, Phys. Rev. Lett. 65, 1838 (1990).

[39] C. Branciard, N. Gisin, and S. Pironio, Characterizing the Nonlocal Correlations Created via Entanglement Swapping, Phys. Rev. Lett. 104, 170401 (2010).

[40] C. Branciard, D. Rosset, N. Gisin, and S. Pironio, Bilocal versus nonbilocal correlations in entanglement-swapping experiments, Phys. Rev. A 85, 032119 (2012).

[41] T. Fritz, Beyond Bell's theorem: Correlation scenarios, New J. Phys. 14, 103001 (2012).

[42] T. Fritz, Beyond Bell's theorem II: Scenarios with arbitrary causal structure, Comm. Math. Phys. 341, 391 (2016).

[43] A. Tavakoli, P. Skrzypczyk, D. Cavalcanti, and A. Ac'in, Nonlocal correlations in the star-network configuration, Phys. Rev. A 90, 062109 (2014).

[44] R. Chaves, Polynomial Bell Inequalities, Phys. Rev. Lett. 116, 010402 (2016).

[45] D. Poderini, I. Agresti, G. Marchese, E. Polino, T. Giordani, A. Suprano, M. Valeri, G. Milani, N. Spagnolo, and G. Carvacho et al., Experimental violation of $n$-locality in a star quantum network, Nat. Commun. 11, 1 (2020).

[46] D. Rosset, C. Branciard, T. J. Barnea, G. Putz, N. Brunner, and N. Gisin, Nonlinear Bell Inequalities Tailored for Quantum Networks, Phys. Rev. Lett. 116, 010403 (2016).

[47] F. Andreoli, G. Carvacho, L. Santodonato, R. Chaves, and F. Sciarrino, Maximal qubit violation of $n$-locality inequalities in a star-shaped quantum network, New J. Phys. 19, 113020 (2017).

[48] R. Chaves, G. Carvacho, I. Agresti, V. Di Giulio, L. Aolita, S. Giacomini, and F. Sciarrino, Quantum violation of an instrumental test, Nat. Phys. 14, 291 (2018).

[49] M.-O. Renou, E. Baumer, S. Boreiri, N. Brunner, N. Gisin, and S. Beigi, Genuine Quantum Nonlocality in the Triangle Network, Phys. Rev. Lett. 123, 140401 (2019).

[50] J. Pearl, Causality (Cambridge university press, Cambridge, United Kingdom, 2009). 
[51] There are actually six distinct latent variable causal structures that faithfully imply the no-signaling constraints (see Ref. [4, Fig. 24]). Those six of those causal structures, however, comprise a single observational equivalence class, as per Evans [80]. That is, all six causal structures imply the same Bell inequalities.

[52] J. P. Jarrett, On the physical significance of the locality conditions in the Bell arguments, Noûs, 569 (1984).

[53] A. A. Fedotov, P. Harremoes, and F. Topsoe, Refinements of Pinsker's inequality, Trans. Info. Theo. 49, 1491 (2003).

[54] L. K. Shalm, E. Meyer-Scott, B. G. Christensen, P. Bierhorst, M. A. Wayne, M. J. Stevens, T. Gerrits, S. Glancy, D. R. Hamel, and M. S. Allman et al., Strong LoopholeFree Test of Local Realism, Phys. Rev. Lett. 115, 250402 (2015).

[55] M. Giustina, M. A. Versteegh, S. Wengerowsky, J. Handsteiner, A. Hochrainer, K. Phelan, F. Steinlechner, J. Kofler, J.-A. Larsson, and C. Abell'an et al., Significant-LoopholeFree Test of Bell's Theorem with Entangled Photons, Phys. Rev. Lett. 115, 250401 (2015).

[56] B. Hensen, H. Bernien, A. E. Dr'eau, A. Reiserer, N. Kalb, M. S. Blok, J. Ruitenberg, R. F. Vermeulen, R. N. Schouten, C. Abell'an, W. Amaya, V. Pruneri, M. W. Mitchell, M. Markham, D. J. Twitchen, D. Elkouss, S. Wehner, T. H. Taminiau, and R. Hanson, Loophole-free Bell inequality violation using electron spins separated by 1.3 kilometres, Nature 526, 682 (2015).

[57] G. Carvacho, F. Andreoli, L. Santodonato, M. Bentivegna, R. Chaves, and F. Sciarrino, Experimental violation of local causality in a quantum network, Nat. Commun. 8, 1 (2017).

[58] R. Chaves, L. Luft, T. O. Maciel, D. Gross, D. Janzing, and B. Schölkopf, Inferring latent structures via information inequalities, ArXiv:1407.2256, (2014).

[59] E. Wolfe, R. W. Spekkens, and T. Fritz, The inflation technique for causal inference with latent variables, J. Causal Inference 7, 0020 (2019).

[60] T. Fritz and R. Chaves, Entropic inequalities and marginal problems, IEEE Trans. Info. Theo. 59, 803 (2012).

[61] R. Chaves, L. Luft, and D. Gross, Causal structures from entropic information geometry and novel scenarios, New J. Phys. 16, 043001 (2014).

[62] R. Chaves, C. Majenz, and D. Gross, Information-theoretic implications of quantum causal structures, Nat. Commun. 6, 1 (2015).

[63] R. W. Yeung, Information Theory and Network Coding (Springer Science \& Business Media, Berlin, Germany, 2008).
[64] H. P. Williams, Fourier's method of linear programming and its dual, Am. Math. Mon. 93, 681 (1986).

[65] D. Collins, N. Gisin, N. Linden, S. Massar, and S. Popescu, Bell Inequalities for Arbitrarily High-Dimensional Systems, Phys. Rev. Lett. 88, 040404 (2002).

[66] M. Ardehali, Bell inequalities with a magnitude of violation that grows exponentially with the number of particles, Phys. Rev. A 46, 5375 (1992).

[67] A. Belinskiui and D. N. Klyshko, Interference of light and Bell's theorem, Physics-Uspekhi 36, 653 (1993).

[68] Formally, the latent projection of Fig. 2 is observationally equivalent to a different DAG wherein $A$ and $X$ are latent-common-cause connected and have identical causal ancestry, as per Ref. [80]. This justifies merging them into a single composite variable without loss of generality.

[69] T. C. Fraser and E. Wolfe, Causal compatibility inequalities admitting quantum violations in the triangle structure, Phys. Rev. A 98, 022113 (2018).

[70] I. Šupić, J.-D. Bancal, and N. Brunner, Quantum Nonlocality in Networks Can Be Demonstrated with an Arbitrarily Small Level of Independence between the Sources, Phys. Rev. Lett. 125, 240403 (2020).

[71] A. Kela, K. Von Prillwitz, J. Åberg, R. Chaves, and D. Gross, Semidefinite tests for latent causal structures, IEEE Trans. Info. Theo. 66, 339 (2020).

[72] J. Åberg, R. Nery, C. Duarte, and R. Chaves, Semidefinite Tests for Quantum Network Topologies, Phys. Rev. Lett. 125, 110505 (2020).

[73] K. Mukherjee, B. Paul, and D. Sarkar, Correlations in n-local scenario, Quant. Info. Proc. 14, 2025 (2015).

[74] S. Popescu and D. Rohrlich, Quantum nonlocality as an axiom, Found. Phys. 24, 379 (1994).

[75] M. Zukowski, A. Zeilinger, M. A. Horne, and A. K. Ekert, Event-Ready-Detectors Bell Experiment via Entanglement Swapping, Phys. Rev. Lett. 71, (1993).

[76] A. Pozas-Kerstjens, R. Rabelo, L. Rudnicki, R. Chaves, D. Cavalcanti, M. Navascu'es, and A. Ac'in, Bounding the Sets of Classical and Quantum Correlations in Networks, Phys. Rev. Lett. 123, 140503 (2019).

[77] G. Moreno et al., Manuscript under preparation.

[78] D. Kédagni and I. Mourifié, Generalized instrumental inequalities testing the instrumental variable independence assumption, Biometrika 107, 661 (2020).

[79] A. Balke and J. Pearl, Bounds on treatment effects from studies with imperfect compliance, J. Am. Stat. Ass. 92, 1171 (1997).

[80] R. J. Evans, Graphs for margins of Bayesian networks, Scand. J. Stat. 43, 625 (2015). 